\title{
Spectral Efficiency and Energy Harvesting in Multi-cell SLIPT Systems
}

\author{
Amr M. Abdelhady, Student Member, IEEE, Osama Amin, \\ Senior Member, IEEE, Basem Shihada, Senior Member, IEEE, and \\ Mohamed-Slim Alouini, Fellow, IEEE
}

\begin{abstract}
In this paper, we study the performance of simultaneous lightwave information and power transfer (SLIPT) systems in a multi-cell indoor scenario. We investigate the energy harvesting and data rate performance of multiple users while meeting the lighting constraints. To this end, we develop optimization frameworks that tune the parameters of transmitters or receivers to improve the SLIPT system performance. Firstly, we model the relationship between tunable lens-based optical receivers concentrator gain and their fields of view. Next, we develop algorithms to maximize the spectral efficiency (SE) considering per-user minimum harvested energy requirements and lightning constraints by controlling the average light emitting diode (LED) excitation current or tuning the optical receivers' fields of view. Then, we study the joint system performance limits of SE and total harvested energy. Towards this aim, we formulate multi-objective optimization problems and propose algorithms to find the best SE energy harvesting tradeoff for both designing strategies. Finally, we present some extensive simulations to explore the benefits of the proposed algorithms comparing with basic benchmarks. Besides, we monitor the effect of changing several system parameters on the two objectives and the behavior of the inherent trade-off between them under both transmitter and receiver sides designing strategies.
\end{abstract}

\section{Index Terms}

Visible light communications, spectral efficiency (SE), energy harvesting, resource allocation, difference of convex/concave (DC) programming, SE - energy harvesting tradeoff, LEDs average current allocation, field of view tuning, Pareto front, multi-objective optimization.

A. M. Abdelhady, O. Amin, B. Shihada and M.-S. Alouini are with with the Computer Electrical, and Mathematical Science and Engineering (CEMSE) Division, King Abdullah University of Science and Technology (KAUST), Thuwal, Makkah Province, Kingdom of Saudi Arabia, E-mail: \{amr.abdelhady, osama.amin, basem.shihada, slim.alouini\}@kaust.edu.sa.

The SE maximization problem by LEDs average current allocation is accepted in IEEE Globecom 2018 [1]. 


\section{INTRODUCTION}

The radio frequency (RF) spectrum over-crowdedness and the fast-paced continuous growth of wireless data traffic have promoted exploiting higher frequency bands in the electromagnetic spectrum [2]. Visible light communication (VLC) has received lots of attention in both academia and industry as it possesses many promising features [3]. VLC systems offer large bandwidth, physical security, great potential in using existing lighting infrastructure, and low cost solutions [4]. These features have nominated VLC to be one of the candidates for next generation wireless technologies [5].

VLC systems can be ubiquitous platforms that offer a multitude of services; they are designed mainly to add a wireless communications support to the fundamental illumination function of the light emitting diodes (LEDs) [6]. In addition, such systems can be very beneficial in providing indoor localization services with a great accuracy [4]. Furthermore, energy can be harvested from light and used to support limited energy devices [7], [8]. Energy consumption at the user equipment has become a very important aspect in new wireless communications networks especially with the evolution of Internet-of-things paradigm [9]. The finiteness of VLC system resources and the co-existence of this multitude of services raise inevitable conflicts between different design objectives. Thus, proper management of system resources becomes of crucial importance in balancing tradeoffs arising between different performance metrics. The achievable rate and energy harvesting tradeoff is analyzed for a single-transmitter and a single-receiver scenario where dynamic power splitting is used to separate energy and information streams in [10]. On the other hand, the tradeoff between illumination and achievable rate is investigated in [11] via multi-objective optimization (MOOP) while imposing an energy harvesting constraint at the receiver end.

Several VLC configurations are designed while considering the light energy harvesting performance [12]-[18]. In [12], Rakia et al. studied a hybrid VLC/RF energy harvesting enabled relaying system and optimized the VLC transmitter DC bias to maximize the average data rate. In the context of multiple users, Zenaidi et al. studied the achievable rate region of a hybrid VLC/RF system where two transmitters are communicating with two designated receivers through an energy harvesting relaying node [13]. In [14], [15], the authors considered a single cell VLC system that serves multiple users and optimized the transmitter resource to maximize the spectral efficiency (SE) with a predefined energy harvesting performance of the multiple users. 
Moreover, Liu at al. demonstrated experimentally the operation of VLC system with a solar cell at the receiver side, which is used to detect the transmitted data and harvest energy in [17]. With the increased research interest in VLC systems possessing energy harvesting capabilities, Diamantoulakis et al. introduced the "SLIPT" term and proposed different operation policies to balance the trade-off between the amount of harvested energy and communications quality of service for a single-user single-cell scenario [16]. Apart from [16], [18], the aforementioned efforts in optimizing VLC systems considered only designing the transmitter side.

Tuning the VLC transmitter side can be done by adapting the transmitted signal, beam direction and beamwidth to optimize the VLC system performance. Nonetheless, tuning the receiver side can introduce additional benefits or give an alternative solution to optimize VLC system performance [19]-[21]. In [19], Al-Ghamdi and Elmirghani studied the effect of adjusting triangular pyramid fly-eye angle diversity receivers' field of view (FoV) on the received signalto-noise ratio and the delay spread. In [20], Park et al. considered a 2x2 multi-input multioutput VLC system and proposed a new receiver structure where a double-sided mirror is used to enhance the received light from one transmitter direction and block it from the other aiming at channel capacity improvement. In [21], Chen et al. proposed and optimized an anglediversity based receiver to reduce the signal-to-interference plus noise ratio fluctuations at the VLC receivers plane for a multicell VLC indoor system for different LED layouts and diversity combining techniques.

In this paper, we study the performance optimization of a multi-cell SLIPT system that is used to serve multiple users. The multi-cell scenario introduces interference on the served users, which adds a challenge on the SLIPT system performance optimization. The contribution of this work is summarized as follows:

- First, we propose a novel optical receiver structure consisting of an electrically tunable lens that focus the light to asymmetric receiving areas of PD and solar cell to account for the trade-off between energy harvesting and spectral efficiency. Then, we derive the optical concentration of the proposed receiver whose focal length, consequently its FoV, can be controlled by an external voltage.

- Next, we design the multicell SLIPT system to maximize the SE performance whilst accounting for spatial illumination and per-device energy harvesting requirements. For this purpose, we consider two operation strategies, namely transmitter-side tuning (TST) and receiver-side tuning (RST), where the transmitting LEDs excitation current and optical 
receivers FoVs are optimized, respectively.

- After that, we study the joint performance of SE and total energy harvested for the two adopted operation strategies using MOOPs to obtain the best trade-off between the two metrics. The TST MOOP problem is non-convex, so we express it as a difference of convex/concave (DC) programming problem to obtain an inner bound for the Pareto frontier of the objectives space before solving it by successive convex approximation. As for the RST MOOP, we show that it is a discrete optimization problem with a decoupled nature, where each receiver can be tuned separately.

- Finally, we demonstrate the achieved gains by optimized systems compared with equal allocation strategies and compare the losses in SE (energy harvesting) performance when energy harvesting (SE) is optimized. In addition, we study the impact of several VLC system parameters on the SE - energy harvesting trade-off behavior.

The rest of this paper is organized as follows: We first describe the assumed system model throughout this paper in section II. After that, we present the overall system SE maximization when TST and RST are considered in section III. Next, we study the joint overall system SE and total energy transfer capabilities in section IV. We present the conducted simulations to evaluate and compare the proposed optimization strategies in section V. Finally, we highlight the paper findings and conclude it in section VI.

\section{SYSTEM DESCRIPTION}

\section{A. Transmitter model}

Consider an array of $K$ LEDs used to illuminate an indoor environment and operate as a SLIPT system to serve $K$ users or less. We assume that all the LEDs have Lambertian radiation pattern with Lambertian order $m=-\ln (2) / \ln \left(\cos \left(\phi_{\mathrm{a}}\right)\right)$, where $\phi_{\mathrm{a}}$ represents the transmitter half-power beamwidth. The quality of lighting service depends on the system ability to achieve satisfactory illuminance levels, maintain uniformity of illuminance on the objects, render the surrounding environment colors properly (in comparison to sunlight), and minimize the amount of flicker [22] as this factors affect people productivity and might cause eyes stress. In this work, we focus on the illuminance uniformity requirements which imply that illuminance at any point

on the illuminated surface be confined between the minimum and maximum values $\left(E_{\min }, E_{\max }\right.$ respectively). We exploit the fact that radiation pattern is Lambertian, thus, most of the radiated luminous flux of each LED will be confined in a local neighborhood around it. Consequently, we 
define the illuminance lower bound to be the least possible illuminance within a cell coverage area provided by the cell transmitter when all the other transmitters are turned off ${ }^{1}$. As for the upper bound, we approximate it as the sum of the maximum illuminances (located right beneath each LED) of the cluster's transmitters i.e (the contributions of LEDs outside a given cluster transmitters' coverage areas are neglected, and those of the cluster elements are upper bounded). We define the cluster as a maximal set of transmitters that are mutually separated by less than $2 d_{\text {min }}$ inter-distance as shown in Fig. 2 , where $d_{\min }$ is the minimum distance between any two transmitters in the setup. We denote the total number of clusters by $|\Phi|$ in the system, with $\Phi$ being the set of all possible clusters in the system. It can be noticed that $\Phi$ depends mainly on the cells deployment layout, for example, in Fig. 2 it can be noticed that $\Phi=\left\{c_{1}, c_{2}, c_{3}, c_{4}\right\}$, where $c_{1}=\{1,2,4,5\}$ and $c_{4}=\{5,6,8,9\}$. We assume that transmitters are placed on the ceiling in a uniform pattern, as shown in Fig. 1. The LEDs are fed together from the same power source with a limited budget of $P_{\mathrm{M}}$ Watt.

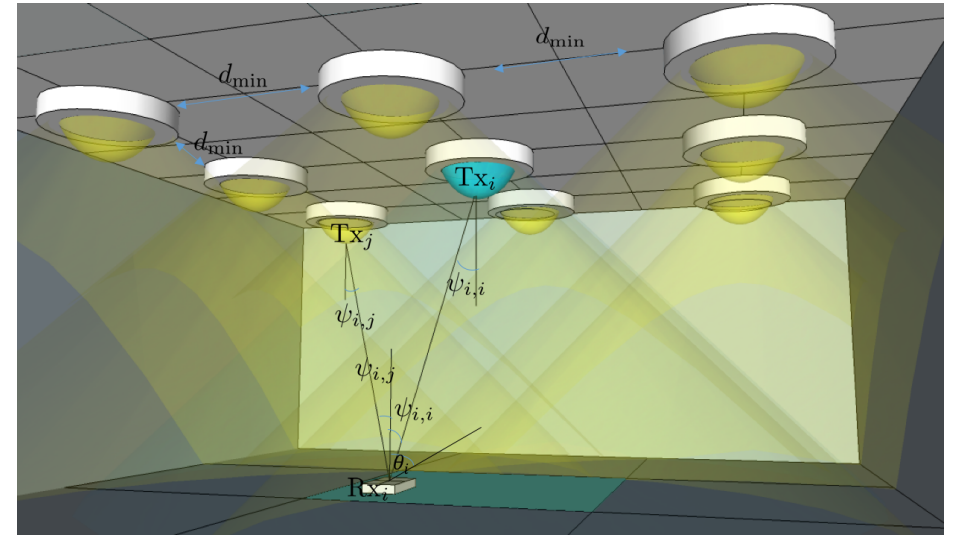

Fig. 1: LED multi-cell deployment layout

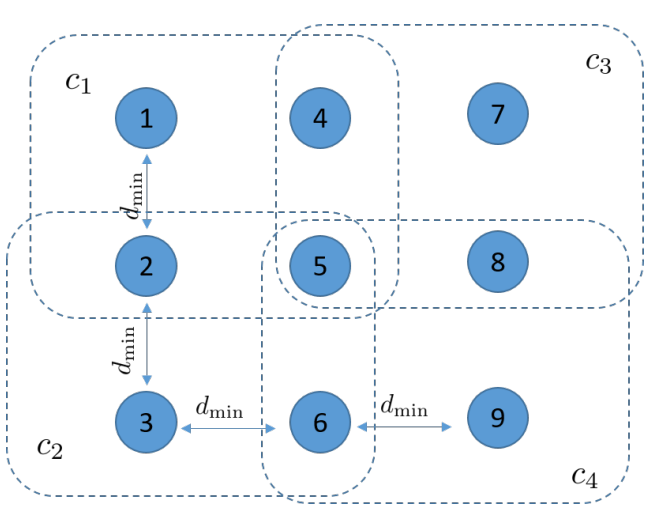

Fig. 2: Transmitters clusters

\section{B. Receiver model}

On the other side, SLIPT receivers are capable of performing dual services: data detection and energy harvesting, which are done in parallel during system operation [23]. We assume the receiving front end is composed of an ideal tunable lens with a circular cross-section of radius $r_{\mathrm{L}}$. The FoV (maximum angle of incidence beyond which the optical receiver cannot sense the

\footnotetext{
${ }^{1}$ In this work, we mean by a cell a construct consisting of a transmitter, its associated coverage area and at most one served receiver.
} 


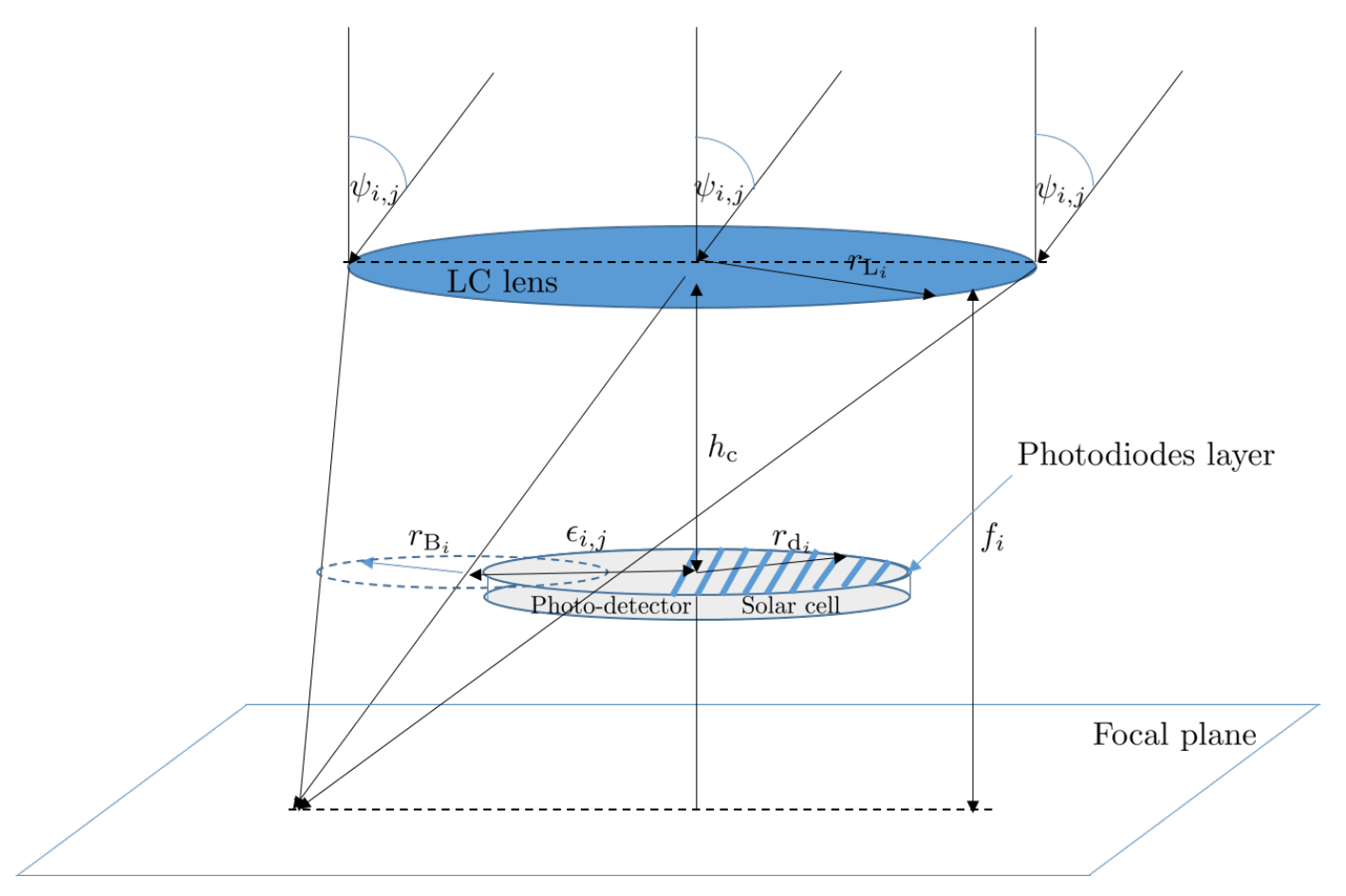

Fig. 3: Tunable FoV Receiver model

incident light) of liquid crystal lens can be controlled electronically [24, Chapter 6] by changing the focal length denoted by $f$. Thus, the optical receiver can increase the power density at its exit aperture compared to the entrance aperture and operate as a tunable optical concentrator. We assume separable detection and energy harvesting circuits are used, where a circular disc of radius $r_{\mathrm{d}}$ and area $A_{\mathrm{D}}$ is divided into two parts of areas $\delta A_{\mathrm{D}}$ and $(1-\delta) A_{\mathrm{D}}$ of a photo-detector and a solar cell, respectively, which are connected to different circuits; as shown in Fig. 3. Using a tunable lens creates a degree of freedom in system operation that can be exploited to either alleviate interference or optimize energy harvesting. Moreover, using a solar cell along with a photo-detector allows system designers to reach performance balance between energy harvesting and communications services due to solar cells limited bandwidth and photo-detectors weak ability in energy scavenging.

The performance of both signal detection and energy harvesting is affected by the concentrator gain, which is upper-bounded by $g_{\mathrm{c}}\left(\theta_{i}\right)=\frac{n_{\mathrm{r}}}{\sin ^{2}\left(\theta_{i}\right)}$ [25], where $n_{\mathrm{r}}$ is the relative refractive index of the concentrator dielectric and $\theta_{i}$ represents receiver $i$ FoV. The above bound becomes exact for the compound parabolic concentrators (non-tunable structure) whose FoV varies inversely with the length of the concentrator, and results in non-practical protruding structures for high 
gain $[26,(4.2)]$. In the following, we derive the concentration gain of the adopted electronic tunable FoV receiver shown in Fig. 3.

1) Concentration gain of a tunable FoV lens: We define $C_{i, j}$ as the concentrator gain of the $i$-th photo-detector in response to transmitter $j$ signal based on the optical concentration gain provided in $[26,2.9]$. Accordingly, we define $C_{i, j}$ as $P_{\mathrm{D}, \mathrm{L}} / P_{\mathrm{D}}$, where $P_{\mathrm{D}, \mathrm{L}}$ is the power density at the optical receiver surface when the lens used, and $P_{D}$ is its counterpart when no lens is used. Assuming that distance between transmitter $j$ and receiver $i$ is large enough to treat the transmitter as a point source, where all incident rays on the lens surface are approximately parallel, having the same angle of incidence $\psi_{i, j}$ (angle between incident ray and the normal to the lens plane) as can be seen in Fig. 3. From geometrical optics and assuming thin ideal collimating lens (aberrations are not considered), parallel incident rays on the lens meet at the intersection of the master ray with the focal plane $[27,1.2 \mathrm{c}]$. It can be seen from the geometry of the receiver that the light rays entering the lens will be collimated into a light cone that intersects the photodiodes plane in a circle of area $A_{\mathrm{B}_{i}}$ and radius $r_{\mathrm{B}_{i}}$, whose center is offset by $\epsilon_{i, j}$ from the detector disc center. By defining the intersection area between the light beam circle in the detection plane and the detector disc to be $A_{\mathrm{I}_{i, j}}$, the transmitted power to the disc can be expressed as $P_{D} A_{\mathrm{L}} \frac{A_{\mathrm{I}_{i, j}}}{A_{\mathrm{B}_{i}}}$ where $A_{\mathrm{L}}$ is the lens cross section area. Hence, the concentrator gain can be expressed as

$$
C_{i, j}=\frac{A_{\mathrm{L}} A_{\mathrm{I}_{i, j}}}{A_{\mathrm{D}} A_{\mathrm{B}_{i}}},
$$

where $A_{\mathrm{B}_{i}}=\pi r_{\mathrm{B}_{i}}^{2}, A_{\mathrm{L}}=\pi r_{\mathrm{L}}^{2}, A_{\mathrm{D}}=\pi r_{\mathrm{d}}^{2}$, and the value of $A_{\mathrm{I}_{i, j}}$ depends on whether $\epsilon_{i, j}>r_{\mathrm{d}}$ or $\epsilon_{i, j}<r_{\mathrm{d}}$ as follows: if $r_{\mathrm{d}}>\epsilon_{i, j}$,

$$
A_{\mathrm{I}_{i, j}}= \begin{cases}\pi r_{\mathrm{B}_{i}}^{2} & 0<r_{\mathrm{B}_{i}} \leq r_{\mathrm{d}}-\epsilon_{i, j}, \\ r_{\mathrm{B}_{i}}^{2} \cos ^{-1}\left(\frac{\epsilon_{i, j}^{2}+r_{\mathrm{B}_{i}}^{2}-r_{\mathrm{d}}^{2}}{2 \epsilon_{i, j} r_{\mathrm{B}_{i}}}\right)+r_{\mathrm{d}}^{2} \cos ^{-1}\left(\frac{\epsilon_{i, j}^{2}+r_{\mathrm{d}}^{2}-r_{\mathrm{B}_{i}}^{2}}{2 \epsilon_{i, j} r_{\mathrm{d}}}\right) & r_{\mathrm{d}}-\epsilon_{i, j}<r_{\mathrm{B}_{i}} \leq r_{\mathrm{d}}+\epsilon_{i, j}, \\ -\frac{1}{2} \sqrt{\left(\left(\epsilon_{i, j}+r_{\mathrm{B}_{i}}\right)^{2}-r_{\mathrm{d}}^{2}\right)\left(r_{\mathrm{d}}^{2}-\left(\epsilon_{i, j}-r_{\mathrm{B}_{i}}\right)^{2}\right)}, & \\ \pi r_{\mathrm{d}}^{2} & r_{\mathrm{d}}<r_{\mathrm{B}_{i}}\end{cases}
$$


otherwise,

$$
A_{\mathrm{I}_{i, j}}= \begin{cases}0 & r_{\mathrm{B}_{i}}<\epsilon_{i, j}-r_{\mathrm{d}} \\ r_{\mathrm{d}}^{2} \cos ^{-1}\left(\frac{\epsilon_{i, j}^{2}+r_{\mathrm{d}}^{2}-r_{\mathrm{B}_{i}}^{2}}{\epsilon_{i, j} r_{\mathrm{d}}}\right)+r_{\mathrm{B}_{i}}^{2} \cos ^{-1}\left(\frac{\epsilon_{i, j}^{2}+r_{\mathrm{B}_{i}}^{2}-r_{\mathrm{d}}^{2}}{2 \epsilon_{i, j} r_{\mathrm{B}_{i}}}\right) & \epsilon_{i, j}-r_{\mathrm{d}}<r_{\mathrm{B}_{i}} \leq \epsilon_{i, j}+r_{\mathrm{d}}, \\ -\frac{1}{2} \sqrt{\left(\left(\epsilon_{i, j}+r_{\mathrm{d}}\right)^{2}-r_{\mathrm{B}_{i}}^{2}\right)\left(r_{\mathrm{B}_{i}}^{2}-\left(\epsilon_{i, j}-r_{\mathrm{d}}\right)^{2}\right)}, & \\ \pi r_{\mathrm{d}}^{2} & \epsilon_{i, j}+r_{\mathrm{d}} \leq r_{\mathrm{B}_{i}},\end{cases}
$$

By following the geometry of the setup it can be noticed that $\epsilon_{i, j}=h_{\mathrm{c}} \tan \left(\psi_{i, j}\right)$, and $r_{\mathrm{B}_{i}}=$ $r_{\mathrm{L}}\left(1-\frac{h_{\mathrm{c}}}{f_{i}}\right)$. It can be seen clearly that if $r_{\mathrm{B}_{i}}+r_{\mathrm{d}}<\epsilon_{i, j}$, there will be no intersection between the light beam circle and the detector circle. Consequently, $r_{\mathrm{B}_{i}}=h \tan \left(\theta_{i}\right)-r_{\mathrm{d}}$, which indicates one-to-one correspondence between $r_{\mathrm{B}_{i}}$ and $\theta_{i}$, and $\tan \left(\theta_{i}\right)=\frac{r_{\mathrm{L}}\left(1-\frac{h_{\mathrm{c}}}{f_{i}}\right)+r_{\mathrm{d}}}{h_{\mathrm{c}}}$, which imposes limits on the obtainable FoV of the optical receiver such that $\tan ^{-1}\left(\frac{r_{\mathrm{d}}}{h_{\mathrm{c}}}\right) \leq \theta_{i} \leq \tan ^{-1}\left(\frac{r_{\mathrm{d}}+r_{\mathrm{L}}}{h_{\mathrm{c}}}\right)$ as $h_{\mathrm{c}} \leq f$. Due to the inherent complexity of the exact concentration ratio expression, we upper bound $A_{\mathrm{I}_{i, j}}$ by $\min \left(A_{\mathrm{B}_{i}}, A_{\mathrm{D}}\right)$ to obtain the following upper bound on $C_{i, j}$

$$
C_{i, j} \leq \tilde{C}_{i, j}=\frac{A_{\mathrm{L}}}{A_{\mathrm{D}}} \frac{\min \left(r_{\mathrm{B}_{i}}^{2}, r_{\mathrm{d}}^{2}\right)}{r_{\mathrm{B}_{i}}^{2}} \mathbf{1}\left(r_{\mathrm{B}_{i}}+r_{\mathrm{d}} \geq \epsilon_{i, j}\right),
$$

where $\mathbf{1}(x)=1$ if $x \geq 0$ and $\mathbf{1}(x)=0$ if $x<0$.

The previous expression can be reformulated in terms of $\theta_{i}$ and $\psi_{i, j}$ as

$$
\tilde{C}_{i, j}=\frac{A_{\mathrm{L}}}{A_{\mathrm{D}}} \min \left(1, \frac{1}{\left(\frac{h_{\mathrm{c}}}{r_{\mathrm{d}}} \tan \left(\theta_{i}\right)-1\right)^{2}}\right) \mathbf{1}\left(\theta_{i} \geq \psi_{i, j}\right)=f_{\mathrm{c}}\left(\theta_{i}\right) \mathbf{1}\left(\theta_{i} \geq \psi_{i, j}\right) .
$$

\section{Channel model and Performance Metrics}

The $K$ LEDs array creates a multi-cell VLC setup where each user is exposed to interference from at most $K-1$ undesired transmitted streams. We assume perfect knowledge of channel state information at the transmitters. The received signal at the $i$-th user photo-detector can be expressed as

$$
y_{i}=\sum_{j=1}^{K} h_{i, j}^{\mathrm{d}} s_{j}+n_{i},
$$

where $s_{i}$ is the current intensity used to transmit a symbol to the $i$-th user by the $j$-th transmitter, $h_{i, j}^{\mathrm{d}}$ is the VLC channel gain coefficient between the $j$-th LED driving current and the $i$-th photodetector generated current, and $n_{i}$ represents the $i$-th receiver noise which is modeled as zero 
mean additive white Gaussian noise with variance $\sigma_{n}^{2}$. The direct link that represents the intended transmission established between the transmitter and its corresponding user has the same indices, i.e., $h_{i, i}^{\mathrm{d}}$. Similarly, $h_{i, j}^{\mathrm{e}}$ is defined as the channel gain coefficient between the $j$-th average LED driving current and the $i$-th solar cell generated current $I_{i}$.

All links are assumed to have line-of-sight existence with neglected reflected components. We adopt the channel model in [25] with the assumption of horizontal orientation for the transmitters and receivers where the channel gains $h_{i, j}^{\mathrm{d}}, h_{i, j}^{\mathrm{e}}$ between transmitter $j$ and receiver $i$ depend on the distance between them $d_{i, j}$, and are upper bounded by

$$
\begin{aligned}
& h_{i, j}^{\mathrm{d}}=\tilde{C}_{i, j}\left(\theta_{i}, \psi_{i, j}\right) \tilde{h}_{i, j}^{\mathrm{d}}, \\
& h_{i, j}^{\mathrm{e}}=\tilde{C}_{i, j}\left(\theta_{i}, \psi_{i, j}\right) \tilde{h}_{i, j}^{\mathrm{e}},
\end{aligned}
$$

where $\tilde{h}_{i, j}^{\mathrm{d}}$ is defined as

$$
\begin{aligned}
& \tilde{h}_{i, j}^{\mathrm{d}}=\frac{\eta_{\phi}}{\eta_{\mathrm{r}}} \frac{(m+1) R_{\mathrm{PD}}^{\mathrm{d}} \delta A_{\mathrm{D}}}{2 \pi d_{i, j}^{2}} \cos ^{m+1}\left(\psi_{i, j}\right), \\
& \tilde{h}_{i, j}^{\mathrm{e}}=\frac{\eta_{\phi}}{\eta_{\mathrm{r}}} \frac{(m+1) R_{\mathrm{PD}}^{\mathrm{e}}(1-\delta) A_{\mathrm{D}}}{2 \pi d_{i, j}^{2}} \cos ^{m+1}\left(\psi_{i, j}\right),
\end{aligned}
$$

where $\eta_{\phi}$ represents the ratio between the LED output luminous flux and its driving current, $\eta_{\mathrm{r}}$ is the LED efficacy (ratio between the output luminous flux and the radiant power), $A_{\mathrm{D}}$ being the effective photo-detector/solar cell area, $R_{\mathrm{PD}}^{\mathrm{d}}$ is the photodiode responsivity of the photo-detector, and $R_{\mathrm{PD}}^{\mathrm{e}}$ is the photodiode responsivity of the solar cell.

In this work, the receivers are assumed to treat interference as noise at detection. The exact expression for VLC channel capacity is not yet available, consequently, we express the system overall SE based on VLC channels in a similar way to [28, Eq. 26], assuming transmitted symbol characteristics at transmitter $i$ of $s_{i} \sim \operatorname{Exp}\left(1 / x_{i}\right)$. We follow similar steps to those done in equations (36-40) of [28], followed by upper bounding the differential entropy of the interference plus noise terms by that of a Gaussian source having same variance [29, Appendix C.2.2] to express $\eta_{\mathrm{SE}}$ as follows

$$
\eta_{\mathrm{SE}}(\boldsymbol{x})=\frac{1}{2} \sum_{i=1}^{K} \log _{2}\left(1+\frac{e}{2 \pi} \frac{\gamma_{i, i} x_{i}^{2}}{1+\sum_{j=1, j \neq i}^{K} \gamma_{i, j} x_{j}^{2}}\right),
$$

where $\boldsymbol{x}=\left\{x_{i}\right\}_{i=1}^{K}, x_{i}=\mathbb{E}\left\{s_{i}\right\}$ and $\gamma_{i, j}$ is the VLC channel-to-noise ratio of the link between the $j$-th transmitter and the $i$-th receiver defined as $\gamma_{i, j}=h_{i, j}^{\mathrm{d}}{ }^{2} / \sigma_{n}^{2} \forall i, j$. Furthermore, each 
receiver can harvest energy from different transmitters that has line of sight with the receiver, i.e., non-zero channel gain. The total harvested power by the $i$-th receiver is expressed based on the computations in $[30,2.5]$ as

$$
P_{\mathrm{H}, i}=0.75 \mathbb{E}\left\{I_{i}\right\} V_{\mathrm{t}} \ln \left(1+\mathbb{E}\left\{I_{i}\right\} / I_{\mathrm{o}}\right)
$$

where $\mathbb{E}\{$.$\} represents the expectation operator, V_{\mathrm{t}}$ is the thermal voltage, $I_{\mathrm{o}}$ is the dark saturation current of the solar cell photodiode and $I_{i}$ represents the current generated in receiver $i$ solar cell, which can be expressed in terms of the LEDs excitation currents as

$$
I_{i}=\sum_{j=1}^{K} h_{i, j}^{\mathrm{e}} s_{j},
$$

(10) follows directly by substituting in [30, Eq. 2.21 ], with fill factor of 0.75 , and $I_{\mathrm{SC}}=\mathbb{E}\left\{I_{i}\right\}$.

The average total power used by the transmitter LEDs to deliver all the required services can be approximated by $\mathbb{E}\left\{\kappa \sum_{i=1}^{K} s_{i}^{2}\right\}=2 \kappa \sum_{i=1}^{K} x_{i}^{2}$, where $\kappa$ represents the equivalent resistance of the LEDs circuit.

\section{SPECTRAL EFFICIENCY MAXIMIZATION}

In this section, we optimize the system parameters to improve the communication performance in terms of SE while satisfying acceptable energy harvesting performance at the receivers side. Designing such a multiple user SLIPT system in an interference-based scenario is a challenging task especially that the interference deteriorates the SE while improves the energy harvesting performance.

In the following, we propose two design strategies to maximize the SE performance when individual energy harvesting requirements are imposed. The first strategy is based on the transmitter side design where the LEDs' average currents are tuned while the second is based on the receiver side design where the receivers' FoVs are adjusted.

\section{A. Transmitter side tuning strategy}

The TST design strategy tunes the average current intensity vector, i.e. $\boldsymbol{x}$, to maximize the total downlink SE. The design should maintain the required illumination level within the required 
range, satisfy the transmitted power budget and support a minimum amount of harvested energy per user. Accordingly, we formulate the following optimization problem:

$$
\begin{array}{ll}
\text { (P1) } \max _{\boldsymbol{x}} & \eta_{\mathrm{SE}}(\boldsymbol{x}) \\
\text { subject to } \quad \mathrm{C} 1: \sum_{i=1}^{K} 2 \kappa x_{i}^{2} \leq P_{\mathrm{M}} & \\
& \mathrm{C} 2: x_{i} \eta_{\mathrm{r}} h_{\min } /\left(A_{\mathrm{D}} R_{\mathrm{PD}}\right) \geq E_{\min } \quad \forall i \\
& \mathrm{C} 3: \sum_{i \in c_{j}} x_{i} \eta_{\mathrm{r}} h_{\max } /\left(A_{\mathrm{D}} R_{\mathrm{PD}}\right) \leq E_{\max } \quad \forall j, \\
& \mathrm{C} 4: P_{\mathrm{H}, i} \geq P_{\mathrm{H}, \mathrm{th}} \quad \forall i,
\end{array}
$$

where $\mathrm{C} 1$ satisfies the transmitter total radiated power budget with $\kappa$ denoting the ratio between the LED electrical power usage and the square of its driving electrical current. The constraints $\mathrm{C} 2$ and $\mathrm{C} 3$ ensure the minimum and maximum allowable illumination levels $E_{\min }$ and $E_{\max }$, respectively, with $h_{\min }$ and $h_{\max }$ being the channel gain at the furthest/nearest point from the transmitter within the cell coverage area and $c_{j}$ representing the $j$-th transmitters cluster. It is worth to mention that ideally $\mathrm{C} 2^{\prime}$ and $\mathrm{C} 3^{\prime}$ to account for spatial illumination requirements,

$\mathrm{C} 2^{\prime}: \quad E_{\min } \leq \min _{\chi \in \mathcal{S}} E(\boldsymbol{\chi} ; \boldsymbol{x})$,

$\mathrm{C} 3^{\prime}: \max _{\chi \in \mathcal{S}} E(\chi ; \boldsymbol{x}) \leq E_{\max }$

where $\boldsymbol{\chi}=\left[\begin{array}{ll}x_{\mathrm{p}} & y_{\mathrm{p}}\end{array}\right]^{\mathrm{T}}$ is the position vector of the test point, and $E(\boldsymbol{\chi} ; \boldsymbol{x})$ is the illuminance at the test point and $\mathcal{S}$ represents the set of all points lying in the receiving plane covered by the transmitters, $E(\boldsymbol{\chi} ; \boldsymbol{x})$ is expressed as

$$
E(\boldsymbol{\chi} ; \boldsymbol{x})=\sum_{j=1}^{K} \frac{(m+1) \eta_{\phi} x_{j}}{2 \pi\left(\left(x_{\mathrm{p}}-x_{j, \mathrm{p}}\right)^{2}+\left(y_{\mathrm{p}}-y_{j, \mathrm{p}}\right)^{2}\right)}\left(\frac{\Delta}{\sqrt{\Delta^{2}+\left(x_{\mathrm{p}}-x_{j, \mathrm{p}}\right)^{2}+\left(y_{\mathrm{p}}-y_{j, \mathrm{p}}\right)^{2}}}\right)^{m+1} .
$$

, where $x_{j, \mathrm{p}}$ and $y_{j, \mathrm{p}}$ represent the $\mathrm{x}$-coordinate and the $\mathrm{y}$-coordinates of the $j$-th transmitter, respectively, and $\Delta$ represents vertical separation between the ceiling and receivers plane. However, due to the inherent complications incurred when $\mathrm{C} 2{ }^{\prime}$ and $\mathrm{C} 3^{\prime}$ are considered, $\mathrm{C} 2$ and C3 are used as a tractable alternative. C4 is used to guarantee minimum amount of harvested 
energy by each user. Interestingly, $P_{\mathrm{H}, i}$ is positive and monotonically increasing with respect to $\sum_{j=1}^{K} h_{i, j}^{\mathrm{e}} x_{j}$, thus we can rewrite $\mathrm{C} 4$ equivalently as

$$
\sum_{j=1}^{K} h_{i, j}^{\mathrm{e}} x_{j} \geq g^{-1}\left(P_{\mathrm{H}, \mathrm{th}}\right)
$$

where $g^{-1}(x)$ is the inverse function of $g(x)=0.75 V_{\mathrm{t}} x \ln \left(1+x / I_{\mathrm{o}}\right)$.

The non-convexity of this problem is evident as it is not convex generally in any of the optimization variables (can be verified easily by evaluating the second derivative $\eta_{\mathrm{SE}}$ ) with respect to any of the variables and checking that the sign is not negative over the entire feasibility region for any possible values of the constraints constants. To solve this problem, we employ alternate optimization where one of the variables is tuned while the rest are kept fixed, such that the updated solution maintains the feasibility conditions and the overall objective function is always improved. Then, roles are changed in a cyclic order between the variable being optimized and the others till achieving the convergence. Thus, in each iteration of the alternate optimization procedure we need to solve a single variable optimization problem of the following form:

$$
\begin{array}{lll} 
& \max _{x_{a}} & \eta_{\mathrm{SE}}\left(x_{a}\right) \\
& \text { subject to } \quad x_{\min , a} \leq x_{a} \leq x_{\max , a}
\end{array}
$$

where $x_{\min , a}$ is defined as

$x_{\min , a}=\max \left(\frac{E_{\min } R_{\mathrm{PD}}}{\eta_{\phi} h_{\min }}, \frac{g^{-1}\left(P_{\mathrm{H}, \mathrm{th}}\right)-\sum_{j \neq a} x_{j} h_{a, j}}{h_{a, a}}\right)$

while $x_{\max , a}$ is defined as

$x_{\max , a}=\min \left(\sqrt{\frac{P_{\mathrm{M}}}{2 \kappa}-\sum_{i \neq a} x_{i}^{2}}, \frac{R_{\mathrm{PD}} E_{\max }}{\eta_{\phi} h_{\max }}-\sum_{\substack{i \in c_{a} \\, i \neq a}} x_{i}\right)$.

The value of $x_{\min , a}$ is imposed by the virtue of $\mathrm{C} 2$ and $\mathrm{C} 4$, while the value of $x_{\max , a}$ is controlled by $\mathrm{C} 1$ and $\mathrm{C} 3$. As for $\eta_{\mathrm{SE}}\left(x_{a}\right)$, it is defined as

$$
\eta_{\mathrm{SE}}\left(x_{a}\right)=\frac{1}{2 \ln (2)}\left(\sum_{i=1}^{K} \ln \left(1+\sum_{j \neq a} \tilde{\gamma}_{i, j} x_{j}^{2}+\tilde{\gamma}_{i, a} x_{a}^{2}\right)-\sum_{\substack{i=1, i \neq a}}^{K} \ln \left(1+\sum_{\substack{j=1, j \notin\{i, a\}}}^{K} \tilde{\gamma}_{i, j} x_{j}^{2}+\tilde{\gamma}_{i, a} x_{a}^{2}\right)\right),
$$

where $\tilde{\gamma}_{i, j}=\gamma_{i, j} \forall i \neq j, \tilde{\gamma}_{i, i}=\frac{e}{2 \pi} \gamma_{i, i} \forall i$.

It can be noticed that $\eta_{\mathrm{SE}}\left(x_{a}\right)$ represents a summation of functions in the following form: $\ln \left(\alpha+\gamma x^{2}\right)$ and $-\ln \left(\beta+\gamma x^{2}\right)$ where both of them can be decomposed into a difference of 
two concave functions of the following forms $-\rho / 2 x^{2}-\left(-\rho / 2 x^{2}-\ln \left(\alpha+\gamma x^{2}\right)\right)$ and $-\bar{\rho} / 2 x^{2}-$ $\left(-\bar{\rho} / 2 x^{2}+\ln \left(\beta+\gamma x^{2}\right)\right)$ respectively, with $\rho \geq \frac{\gamma}{4 \alpha}$ and $\bar{\rho} \geq 2 \gamma$. These decompositions can be derived in a very similar way to the steps followed in [31, Eq. 3 -12]. Therefore, (P2) is a DC programming problem that can be solved by successive convex approximation (SCA) where the following optimization problem is solved in each SCA iteration:

$$
\begin{array}{lll} 
& \max _{x_{a}} & \tilde{\eta}_{\mathrm{SE}}\left(x_{a}\right) \\
& \text { subject to } & x_{\min , a} \leq x_{a} \leq x_{\max , a}
\end{array}
$$

where $\tilde{\eta}_{\mathrm{SE}}\left(x_{a}\right)$ is written as

$$
\begin{aligned}
& \tilde{\eta}_{\mathrm{SE}}\left(x_{a}\right)= \\
& \frac{1}{2 \ln (2)} \sum_{i=1}^{K}\left(-\frac{\rho_{a, i} x_{a}^{2}}{2}+\left(\frac{\rho_{a, i} x_{a, 0}^{2}}{2}+\ln \left(\Gamma_{a}+\tilde{\gamma}_{i, a} x_{a, 0}^{2}\right)+\left(\rho_{a, i} x_{a, 0}+\frac{2 \tilde{\gamma}_{i, a} x_{a, 0}}{\Gamma_{a}+\tilde{\gamma}_{i, a} x_{a, 0}^{2}}\right)\left(x_{a}-x_{a, 0}\right)\right)\right) \\
& +\frac{1}{2 \ln (2)} \sum_{i=1, i \neq a}^{K}\left(-\frac{\bar{\rho}_{a, i} x_{a}^{2}}{2}+\left(\frac{\bar{\rho}_{a, i} x_{a, 0}^{2}}{2}-\ln \left(\Gamma_{a, i}+\tilde{\gamma}_{i, a} x_{a, 0}^{2}\right)+\left(\bar{\rho}_{a, i} x_{a, 0}-\frac{2 \tilde{\gamma}_{i, a} x_{a, 0}}{\Gamma_{a, i}+\tilde{\gamma}_{i, a} x_{a, 0}^{2}}\right)\left(x_{a}-x_{a, 0}\right)\right)\right),
\end{aligned}
$$

with $\rho_{a, i}=\frac{\tilde{\gamma}_{i, a}}{4 \Gamma_{a}}, \bar{\rho}_{a, i}=2 \tilde{\gamma}_{i, a}, \Gamma_{a}=1+\sum_{j \neq a} \tilde{\gamma}_{i, j} x_{j}^{2}, \Gamma_{a, i}=1+\sum_{j \neq a, j \neq i} \tilde{\gamma}_{i, j} x_{j}^{2}$ and $x_{a, 0}$ is the solution obtained in the previous SCA iteration. It can be noticed that the objective function is a concave quadratic polynomial in $x_{a}$, hence, the solution of the previous problem is the just the projection of the unconstrained version of the problem on the feasibility interval, which can be expressed in a closed form as

$x_{a}^{*}=\max \left(\min \left(x_{\mathrm{cr}}, x_{\max , a}\right), x_{\min , a}\right)$,

where $x_{\mathrm{cr}}$ is found to be

$x_{\mathrm{cr}}=\frac{x_{a, 0} \sum_{i=1}^{K}\left(\rho_{a, i}+\frac{2 \tilde{\gamma}_{i, a}}{\Gamma_{a}+\tilde{\gamma}_{i, a} x_{a, 0}^{2}}+\bar{\rho}_{a, i}-\frac{2 \tilde{\gamma}_{i, a}}{\Gamma_{a, i}+\tilde{\gamma}_{i, a} x_{a, 0}^{2}}\right)}{\sum_{i=1}^{K} \rho_{a, i}+\sum_{i \neq a} \bar{\rho}_{a, i}}$.

Based on the previous discussion, our proposed solution procedure for $(\mathrm{P} 1)$ proceeds as shown in Algorithm I. At first, a set of initial solutions $\mathcal{F}$ is created using the feasibility problem (P4) solution along with two equal allocation vectors, that will be discussed later (they are added to $\mathcal{F}$ if they satisfy the energy harvesting requirement) as indicated in lines (2-8). Afterwards, (P1) is solved through the iterative procedure starting with different elements from $\mathcal{F}$, then the best 


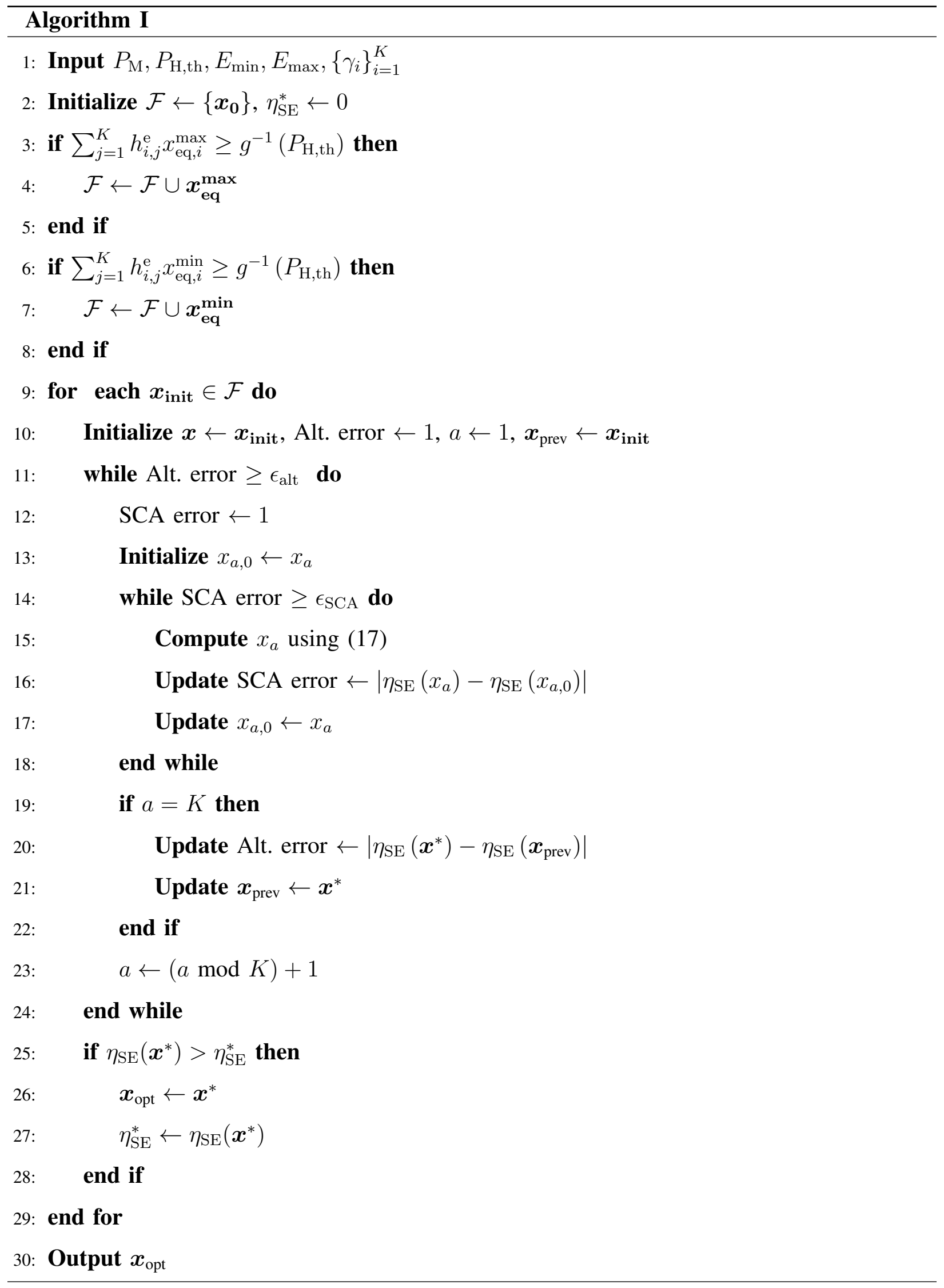


solution is chosen. Solving (P1) is done over two nested phases: in the outer phase (lines 1124) (P1) is solved iteratively by alternate optimization where each element in $\boldsymbol{x}$ is optimized separately where the rest of the elements are kept fixed by solving a customized version of (P2). In the inner phase (lines 14-18), the solution of each version of (P2) is obtained by successively solving different SCA-versions of (P3) using the closed form expression given by (17). Algorithm I convergence to a better solution than the initial one is guaranteed by two facts; the first is that the inner phase converges to a stationary point solution for (P2) as it is a DC programming problem solved using SCA, alternatively known as convex concave procedure as in [32]. The second fact is that Algorithm I terminates if the prospective alternate optimization step does not improve the objective value.

By defining the worst case number of alternating optimization iterations as $I_{\text {Alt. }}$, worst case number of iterations of the SCA procedure as $I_{\mathrm{SCA}}$, the overall complexity of Algorithm $\mathrm{I}$ is in the order of $3 I_{\mathrm{Alt}} I_{\mathrm{S} C A} K$ operations as specified in (17). The factor of three appearing represents the possibility that the alternate optimization procedure will be executed for three feasible initial points.

1) Feasibility problem: Before solving the SE maximization problem, we need to examine the problem feasibility. A simple way to do so is to solve the following simplified optimization problem:
(P4) $\min _{x}$
$2 \kappa \sum_{i=1}^{K} x_{i}^{2}$
subject to $\mathrm{C} 2, \mathrm{C} 3, \mathrm{C} 4$.

After solving (P4), it can be deduced that $(\mathrm{P} 1)$ is infeasible if $(\mathrm{P} 4)$ is infeasible or the optimal value of $(\mathrm{P} 4)$ exceeds $P_{\mathrm{M}}$. $(\mathrm{P} 4)$ is a convex quadratic programming problem, thus we can solve it using convex optimization subroutines such as MATLAB CVX package [33].

2) Choosing initial point: Since the algorithm performance varies with the starting point, so we solve the problem using three possible initial points and take the best solution. The possible intial points are:

1) $\boldsymbol{x}_{\mathrm{eq}}^{\min }: x_{\mathrm{eq}, i}^{\min }=\frac{E_{\min } R_{\mathrm{PD}}}{\eta_{\phi} h_{\min }} \forall i$, which is the equal allocation associated with the minimum illumination constraint. This proposed starting point gives a good solution to the dense cell deployment scenario where it is desirable to reduce the interference signal. 
2) $\boldsymbol{x}_{\mathrm{eq}}^{\max }: x_{\mathrm{eq}, i}^{\max }=\min \left(\sqrt{\frac{P_{\mathrm{M}}}{2 \kappa K}}, \frac{E_{\max } R_{\mathrm{PD}}}{\eta_{\phi} h_{\max } \max _{j}\left|c_{j}\right|}\right) \forall i$, which is the equal allocation associated with the maximum illuminance constraint ${ }^{2}$. This initial point gives a good solution to the sparse cell deployment scenario, where it is beneficial to have a strong signal to improve SE.

3) $x_{0}$ : the solution of $(\mathbf{P} 4)$, which becomes important when the two previous initial points do not satisfy $\mathrm{C} 4$.

\section{B. Receiver side tuning strategy}

In order to have a ubiquitous vision on the performance limits of SLIPT systems, we study the receivers side optimization with the objective of overall system SE maximization under individual receivers energy harvesting constraints. This study is motivated by the electrical tunable spatial selectivity (FoV) offered by the considered optical receiver structure, which promotes for high interference reduction capability.

The problem of SE maximization for a given average allocated current intensity at the transmitters side where each receiver should optimize its FoV is written as:

$$
\begin{array}{cl}
\max _{\boldsymbol{\theta}} & \frac{1}{2 \ln (2)} \sum_{i=1}^{K} \ln \left(1+\frac{\bar{\gamma}_{i, i} x_{i}^{2} f_{\mathrm{c}}^{2}\left(\theta_{i}\right) \mathbf{1}\left(\theta_{i} \geq \psi_{i, i}\right)}{1+\sum_{j=1, j \neq i}^{K} \bar{\gamma}_{i, j} x_{j}^{2} f_{\mathrm{c}}^{2}\left(\theta_{i}\right) \mathbf{1}\left(\theta_{i} \geq \psi_{i, j}\right)}\right) \\
\text { subject to } \quad \mathrm{C} 4: f_{\mathrm{c}}\left(\theta_{i}\right) \sum_{j=1}^{K} \tilde{h}_{i, j}^{\mathrm{e}} \mathbf{1}\left(\theta_{i} \geq \psi_{i, j}\right) x_{j} \geq g^{-1}\left(P_{\mathrm{H}, \mathrm{th}}\right) \quad \forall i, \\
\mathrm{C} 5: \theta_{\min } \leq \theta_{i} \leq \theta_{\max } \quad \forall i,
\end{array}
$$

where $\bar{\gamma}_{i, j}=\left(\tilde{h}_{i, j}^{\mathrm{d}}\right)^{2} / \sigma_{n}^{2} \forall i \neq j, \bar{\gamma}_{i, i}=\frac{e}{2 \pi}\left(\tilde{h}_{i, i}^{\mathrm{d}}\right)^{2} / \sigma_{n}^{2}$. In this problem formulation, we do not consider the $\mathrm{C} 1-\mathrm{C} 3$ constraints as in (P1) because FoVs do not affect them. C5 is imposed here to account for the physical limitations of the optical receiving device as explained in section II. It can be observed easily that both the objective function and the constraints are decoupled in the optimization variables. Thus, it is equivalent to optimize the FoV of each of the $K$ receivers separately according to the following problem:

\footnotetext{
${ }^{2}\left|c_{i}\right|$ represents the cardinality of the set $c_{i}$ (number of transmitters in cluster $i$ which is constant due to uniform lattice layout of transmitters and equals 4 for square grid lattice).
} 
$(\overline{\mathbf{P}} 5) \max _{\theta_{i}} \quad \frac{1}{2 \ln (2)} \ln \left(1+\frac{\bar{\gamma}_{i, i} x_{i}^{2} \mathbf{1}\left(\theta_{i} \geq \psi_{i, i}\right)}{\frac{1}{f_{\mathrm{c}}^{2}\left(\theta_{i}\right)}+\sum_{j=1, j \neq i}^{K} \bar{\gamma}_{i, j} x_{j}^{2} \mathbf{1}\left(\theta_{i} \geq \psi_{i, j}\right)}\right)$

subject to $\mathrm{C} 4, \mathrm{C} 5$,

Since the objective function value is zero if $\theta_{i}<\psi_{i, i}$, this problem is equivalent to the following formulation

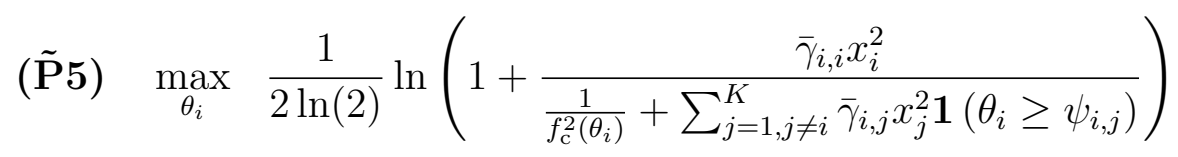

subject to $\quad \mathrm{C} 4, \tilde{\mathrm{C}} 5: \max \left(\theta_{\min }, \psi_{i, i}\right) \leq \theta_{i} \leq \theta_{\max }$,

Since both terms $\frac{1}{f_{\mathrm{c}}^{2}\left(\theta_{i}\right)}$ and $\sum_{j=1, j \neq i}^{K} \bar{\gamma}_{i, j} x_{j}^{2} \mathbf{1}\left(\theta_{i} \geq \psi_{i, j}\right)$ are positive non-decreasing functions of $\theta_{i}$, and $\ln \left(1+\frac{1}{)}\right)$ is a decreasing function for positive arguments, then the objective function is non-increasing in $\theta_{i}$, and consequently the optimal $\theta_{i}$ values is the smallest feasible one.

By focusing on the feasibility region, $\mathrm{C} 4$ particularly, it can be noticed that $f_{\mathrm{c}}\left(\theta_{i}\right) \sum_{j=1}^{K} \tilde{h}_{i, j}^{\mathrm{e}} \mathbf{1}\left(\theta_{i} \geq \psi_{i, j}\right) x_{j} \geq g^{-1}\left(P_{\mathrm{H}, \mathrm{th}}\right)$ is a non-increasing function of $\theta_{i}$ over any interval of this form $\psi_{\pi_{l}^{(i)}} \leq \theta_{i}<\psi_{\pi_{l+1}^{(i)}} \forall l \in\{1, \ldots, K\}$, where $\psi_{\pi_{l}^{(i)}} \leq \psi_{\pi_{j}^{(i)}} \forall l \leq j$. Hence, the optimal value of $\theta_{i}$ lies at one of the angles of incidences that satisfy $\tilde{\mathrm{C}} 5$ or at $\theta_{\min }$ in case $\theta_{\min }$ satisfies $\mathrm{C} 4$ and $\theta_{\min } \geq \psi_{i, i}$. Finally, the optimal $\theta_{i}$ can be expressed as

$$
\theta_{i}^{*}=\min _{x \in \mathcal{S}} x, \quad \mathcal{S}=\left\{\max \left(\theta_{\min }, \psi_{i, i}\right)\right\} \cup \mathcal{S}_{2},
$$

where $\mathcal{S}_{2}=\left\{\psi_{i, l} \quad \forall l \in 1, \ldots, K: \sum_{j=1}^{K} \tilde{h}_{i, j}^{\mathrm{e}} f_{\mathrm{c}}\left(\psi_{i, l}\right) \mathbf{1}\left(\psi_{i, l} \geq \psi_{i, j}\right) x_{j} \geq g^{-1}\left(P_{\mathrm{H}, \mathrm{th}}\right), \theta_{\min } \leq\right.$ $\left.\psi_{i, l} \leq \theta_{\max }\right\}$. The computational complexity of solving (P5) is summarized in evaluating whether $\mathrm{C} 4$ is satisfied or not for at most $K$ possible values for each of the $K$ receivers, which in total results in the order of $K^{2}$ operations.

The previously considered SLIPT optimization framework is well suited for systems having priority for maintaining receiving devices battery life time, thus energy harvesting was imposed individually. However, studying the joint performance of both communication and energy harvesting services in order to know the performance limit of both services becomes of crucial importance and is discussed in the following section.

\section{JOINT INFORMATION AND ENERGY TRANSFER PERFORMANCE FRONTIER}

In this section, we investigate the joint performance of both the total information and energy delivered from different LED sources in a multi-cell multi-user VLC network. The conflict 
between both performance metrics arises mainly from the interference which degrades the SE while it improves the harvested energy. To this end, we adopt MOOP to obtain the best tradeoff performance for both TST and RST strategies.

\section{A. Pareto Front Performance of Transmitter Side Tuning Strategy}

There are many ways that can be considered in MOOP as scalarization using linear and nonlinear weights [34], [35]. To obtain a Pareto front for the SE and energy harvesting performance for the TST strategy, we use the weighted sum MOOP, which is a utility function formed by adding the two normalized objective functions each of which being weighted by its importance according to the system operation preferences. Consequently, the optimization problem is defined as follows:

$$
\text { (P6) } \begin{aligned}
\max _{\boldsymbol{x}} & \frac{\alpha}{2 \ln (2) \eta_{\mathrm{SE}}^{\max }}\left(\sum_{i=1}^{K} \ln \left(1+\sum_{j=1}^{K} \tilde{\gamma}_{i, j} x_{j}^{2}\right)-\sum_{i=1}^{K} \ln \left(1+\sum_{j=1, j \neq i}^{K} \tilde{\gamma}_{i, j} x_{j}^{2}\right)\right) \\
& +\frac{1-\alpha}{P_{\mathrm{H}, \mathrm{tot}}^{\max }}\left(0.75 V_{\mathrm{t}} \sum_{i=1}^{K} \sum_{l=1}^{K} h_{i, l}^{\mathrm{e}} x_{l} \ln \left(1+\sum_{j=1}^{K} h_{i, j}^{\mathrm{e}} x_{j} / I_{\mathrm{o}}\right)\right)
\end{aligned}
$$

subject to $\mathrm{C} 1, \mathrm{C} 2, \mathrm{C} 3$,

where $\alpha$ is the tradeoff parameter with $0 \leq \alpha \leq 1$, and $\eta_{\mathrm{SE}}^{\max }, P_{\mathrm{H} \text {,tot }}^{\max }$ are the maximized SE and total harvested power obtained using Algorithm II, respectively. One can show that (P6) is a non-convex problem, by changing the optimization variables to be $\left\{p_{1}, \ldots, p_{K}\right\} \triangleq \boldsymbol{p}$, where $p_{i}=x_{i}^{2} \forall i$. (P6), is reformulated to the following equivalent DC MOOP (for proof please check Appendix A) as

$$
\begin{array}{cl}
\max _{\boldsymbol{p}} & \eta_{\mathrm{MOOP}}(\boldsymbol{p}) \\
\text { subject to } & \tilde{\mathrm{C}} 1: \sum_{i=1}^{K} 2 \kappa p_{i} \leq P_{\mathrm{M}} \\
& \tilde{\mathrm{C}} 2: p_{i} \geq\left(\frac{E_{\min } R_{\mathrm{PD}}}{\eta_{\phi} h_{\min }}\right)^{2} \forall i \\
& \tilde{\mathrm{C}} 3: \sum_{i \in c_{j}} p_{i}^{2}-\left(\sum_{i \in c_{j}} p_{i}^{2}-\sum_{i \in c_{j}} \sqrt{p_{i}}\right) \leq \frac{R_{\mathrm{PD}} E_{\max }}{\eta_{\phi} h_{\max }} \forall j,
\end{array}
$$


where

$\eta_{\mathrm{MOOP}}(\boldsymbol{p})=\frac{\alpha}{2 \ln (2) \eta_{\mathrm{SE}}^{\max }}\left(\sum_{i=1}^{K} \ln \left(1+\sum_{j=1}^{K} \tilde{\gamma}_{i, j} p_{j}\right)-Q(\boldsymbol{p})\right)+\frac{1-\alpha}{P_{\mathrm{H}, \mathrm{tot}}^{\max }} P_{\mathrm{H}, \mathrm{tot}}(\boldsymbol{p})$,

$Q(\boldsymbol{p})=\sum_{i=1}^{K} \ln \left(1+\sum_{j=1, j \neq i}^{K} \tilde{\gamma}_{i, j} p_{j}\right)$

where

$$
P_{\mathrm{H}, \mathrm{tot}}(\boldsymbol{p})=0.75 V_{\mathrm{t}} \sum_{i=1}^{K} \sum_{l=1}^{K} h_{i, l}^{\mathrm{e}} \sqrt{p_{l}} \ln \left(1+\sum_{j=1}^{K} h_{i, j}^{\mathrm{e}} \sqrt{p_{j}} / I_{\mathrm{o}}\right)
$$

$\tilde{\mathrm{C}} 1, \tilde{\mathrm{C}} 2$ represent the equivalent versions of $\mathrm{C} 1, \mathrm{C} 2$ after variables transformation, respectively, and $\tilde{\mathrm{C}} 3$ is the DC reformulated version of C3.

We solve P7 using SCA, where in each iteration the following problem is solved successively,

$$
\frac{\alpha}{2 \ln (2) \eta_{\mathrm{SE}}^{\max }}\left(\sum_{i=1}^{K} \ln \left(1+\sum_{j=1}^{K} \tilde{\gamma}_{i, j} p_{j}\right)-\tilde{Q}(\boldsymbol{p})\right)+\frac{1-\alpha}{P_{\mathrm{H}, \text { tot }}^{\max }} P_{\mathrm{H}, \mathrm{tot}}(\boldsymbol{p})
$$

subject to $\tilde{\mathrm{C}} 1: \sum_{i=1}^{K} 2 \kappa p_{i} \leq P_{\mathrm{M}}$,

$$
\begin{aligned}
& \tilde{\mathrm{C}} 2: p_{i} \geq\left(\frac{E_{\mathrm{min}} R_{\mathrm{PD}}}{\eta_{\phi} h_{\min }}\right)^{2} \forall i \\
& \overline{\mathrm{C}} 3: \sum_{i \in c_{j}}\left(p_{i}^{2}-\left(\bar{p}_{i}^{2}-\sqrt{\bar{p}_{i}}+\left(2 \bar{p}_{i}-\frac{1}{2 \sqrt{\bar{p}_{i}}}\right)\left(p_{i}-\bar{p}_{i}\right)\right)\right) \leq \frac{R_{\mathrm{PD}} E_{\max }}{\eta_{\phi} h_{\max }} \quad \forall j,
\end{aligned}
$$

where $\overline{\mathrm{C}} 3$ represents a convex set approximation for the original non-convex set defined by $\tilde{\mathrm{C}} 3$, and $\tilde{Q}(\boldsymbol{p})=g(\overline{\boldsymbol{p}})+\left(\left.\nabla g\right|_{\overline{\boldsymbol{p}}}\right)^{T}(\boldsymbol{p}-\overline{\boldsymbol{p}})$, such that $\nabla g$ represents the gradient of the function $g($. and the first derivative of $g(\boldsymbol{p})$ is given as

$$
\frac{\partial g}{\partial p_{a}}=\sum_{i=1}^{K} \frac{\gamma_{i, a}}{1+\sum_{j=1}^{K} \tilde{\gamma}_{i, j} p_{j}-\tilde{\gamma}_{i, i} p_{i}}-\frac{\gamma_{a, a}}{1+\sum_{j=1}^{K} \tilde{\gamma}_{a, j} p_{j}-\tilde{\gamma}_{a, a} p_{a}} \quad \forall a \in\{1, \ldots, K\} .
$$

The solution of (P7) proceeds as described in Algorithm II, where at first two initial solutions are selected to enhance chances of reaching an optimal solution. This is well reasoned by the non-convexity of the problem which creates dependency between the final reached solution and the initial starting point, in addition to the iterative gradient-based approach used to solve it. Thus, two feasible easy-to-obtain initial guesses are chosen, where one of them performs well in the dense deployment regime while the other works well in sparse deployment setups. After the initialization step, the Algorithm proceeds by solving (P8) with $\bar{p}$ being the initial solution 
using interior point method. Subsequently, the obtained solution is fed into $\bar{p}$ and (P8) is solved in a cyclic manner till convergence as shown in lines (6-10) of Algorithm II. Finally, the other starting point is used and the whole process is repeated to find the best solution.

As for the computational complexity incurred on executing Algorithm II, it depends on the number of SCA procedure outer iterations $I_{\mathrm{SCA}}^{\mathrm{MOOP}}$, and the complexity of solving the convex optimization problem encountered within each SCA iteration is in the order of $\zeta \max \left(K^{3}, K^{2}(2 K+1+|\Phi|),(2 K+1+|\Phi|)\left(K+K^{2}\right)\right)$ operations, where $\zeta$ is constant between 10 and 100 for most of the practical problems [36]. Consequently the overall complexity is in the order of $2 I_{\mathrm{SCA}}^{\mathrm{MOOP}} \zeta \max \left(K^{3}, K^{2}(2 K+1+|\Phi|),(2 K+1+|\Phi|)\left(K+K^{2}\right)\right)$ operations, where the scalar 2 appearing at the beginning of the complexity expression accounts for running the SCA procedure for two different initial points. The convergence of Algorithm II to a stationary point of (P7) is guaranteed as it solves DC programming problem with convex-concave procedure [32].

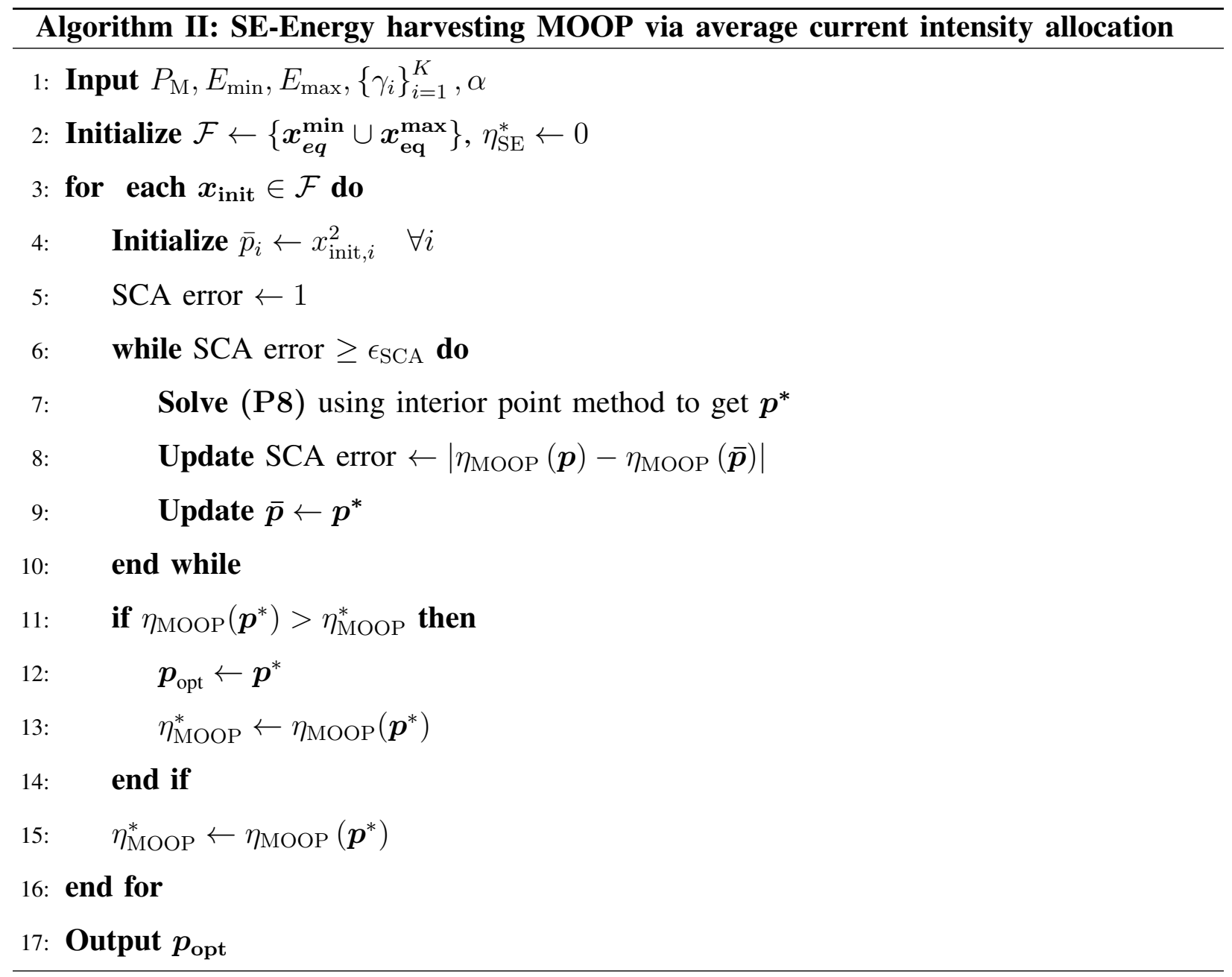




\section{B. Spectral Efficiency - Energy Harvesting Tradeoff Performance for Receiver Side Tuning}

\section{Strategy}

Tuning the FoVs of receivers raises a conflict between the overall system SE and the total harvested energy by the receivers as long as beamforming is not adopted at the transmitters. To find the optimal tradeoff between SE and total harvested energy when receivers' FoVs are tuned, we formulate the following MOOP problem

(P9) $\max _{\theta}$

$$
\begin{gathered}
\frac{\alpha}{2 \ln (2) \eta_{\mathrm{SE}}^{\max , 2}} \sum_{i=1}^{K} \ln \left(1+\frac{\bar{\gamma}_{i, i} x_{i}^{2}}{\frac{1}{f_{c}^{2}\left(\theta_{i}\right)}+\sum_{j=1, j \neq i}^{K} \bar{\gamma}_{i, j} x_{j}^{2} \mathbf{1}\left(\theta_{i} \geq \psi_{i, j}\right)}\right)+\frac{1-\alpha}{P_{\mathrm{H}, \mathrm{tot}}^{\max , 2}} \times \\
\left(\sum_{i=1}^{K} \sum_{j=1}^{K} 0.75 h_{i, j}^{\mathrm{e}} f_{\mathrm{c}}\left(\theta_{i}\right) \mathbf{1}\left(\theta_{i} \geq \psi_{i, j}\right) x_{j} \mathrm{~V}_{\mathrm{t}} \ln \left(1+\sum_{j=1}^{K} h_{i, j}^{\mathrm{e}} f_{\mathrm{c}}\left(\theta_{i}\right) \mathbf{1}\left(\theta_{i} \geq \psi_{i, j}\right) \frac{x_{j}}{I_{o}}\right)\right)
\end{gathered}
$$

subject to $\quad \mathrm{C} 5: \max \left(\theta_{\min }, \psi_{i, i}\right) \leq \theta_{i} \leq \theta_{\max } \quad \forall i$,

where $\eta_{\mathrm{SE}}^{\max , 2}$ and $P_{\mathrm{H} \text {,tot }}^{\max , 2}$ represent the maximum $\mathrm{SE}$ and maximum total harvested power, respectively, obtainable through FoVs tuning.

One can clearly observe from (P9) that there is no coupling between the optimization parameters, neither in the objective function nor in the constraints. Hence, every $\theta_{i}$ can be tuned separately by solving the following optimization problem

(P10) $\max _{\theta_{i}}$

$$
\begin{gathered}
\frac{\alpha}{2 \ln (2) \eta_{\mathrm{SE}}^{\max , 2}} \ln \left(1+\frac{\bar{\gamma}_{i, i} x_{i}^{2}}{\frac{1}{f_{\mathrm{c}}^{2}\left(\theta_{i}\right)}+\sum_{j=1, j \neq i}^{K} \bar{\gamma}_{i, j} x_{j}^{2} \mathbf{1}\left(\theta_{i} \geq \psi_{i, j}\right)}\right)+\frac{1-\alpha}{P_{\mathrm{H}, \mathrm{tot}}^{\max , 2}} \times \\
\left(\sum_{j=1}^{K} 0.75 h_{i, j}^{\mathrm{e}} f_{\mathrm{c}}\left(\theta_{i}\right) \mathbf{1}\left(\theta_{i} \geq \psi_{i, j}\right) x_{j} \mathrm{~V}_{\mathrm{t}} \ln \left(1+\sum_{j=1}^{K} h_{i, j}^{\mathrm{e}} f_{\mathrm{c}}\left(\theta_{i}\right) \mathbf{1}\left(\theta_{i} \geq \psi_{i, j}\right) \frac{x_{j}}{I_{o}}\right)\right)
\end{gathered}
$$

subject to $\mathrm{C} 5: \max \left(\theta_{\min }, \psi_{i, i}\right) \leq \theta_{i} \leq \theta_{\max }$.

By defining the following set of intervals:

$\mathcal{I}=\{\underbrace{\left[0, \psi_{i, \pi_{1}^{(i)}}\right)}_{\mathcal{I}_{0}}, \ldots, \underbrace{\left[\psi_{i, \pi_{\ell}^{(i)}}, \psi_{i, \pi_{\ell+1}^{(i)}}\right)}_{\mathcal{I}_{\ell}}, \ldots, \underbrace{\left[\psi_{i, \pi_{K}^{(i)}}, \infty\right)}_{\mathcal{I}_{K}}\}$, where $\psi_{i, \pi_{\ell}^{(i)}} \leq \psi_{i, \pi_{j}^{(i)}} \forall \ell \leq j$, and $\ell \in\{1, \ldots, K-1\}$. Solving $(\mathbf{P} 10)$ is equivalent to solving $K+1$ problems of the form 
$\begin{aligned}\left(\mathbf{P 1 0}^{\prime}\right) \max _{\theta_{i}} \quad & \frac{\alpha}{2 \ln (2) \eta_{\mathrm{SE}}^{\max , 2}} \ln \left(1+\frac{\bar{\gamma}_{i, i} x_{i}^{2}}{\frac{1}{f_{\mathrm{c}}^{2}\left(\theta_{i}\right)}+\sum_{j=1, j \neq i}^{K} \bar{\gamma}_{i, j} x_{j}^{2} \mathbf{1}\left(\theta_{i} \geq \psi_{i, j}\right)}\right)+\frac{1-\alpha}{P_{\mathrm{H}, \mathrm{tot}}^{\max , 2} \times} \\ & \left(\sum_{j=1}^{K} 0.75 h_{i, j}^{\mathrm{e}} f_{\mathrm{c}}\left(\theta_{i}\right) \mathbf{1}\left(\theta_{i} \geq \psi_{i, j}\right) x_{j} \mathrm{~V}_{\mathrm{t}} \ln \left(1+\sum_{j=1}^{K} h_{i, j}^{\mathrm{e}} f_{\mathrm{c}}\left(\theta_{i}\right) \mathbf{1}\left(\theta_{i} \geq \psi_{i, j}\right) \frac{x_{j}}{I_{o}}\right)\right)\end{aligned}$

subject to $\mathrm{C}^{\prime}: \theta_{i} \in\left[\max \left(\theta_{\min }, \psi_{i, i}\right), \theta_{\max }\right] \cap \mathcal{I}_{l} \quad, l \in\{0, \ldots, K\}$.

, then selecting the solution that maximizes the objective function of (P10).

By exploiting $\mathrm{C} 5^{\prime}$ which enforces the thresholds of the indicator functions to lie on the edges of the feasibility region, the objective function of $(\mathrm{P} 10)$ can be re-written by omitting the indicator functions to re-express $\left(\mathrm{P}^{\prime} \mathbf{0}^{\prime}\right)$ as

$$
\begin{aligned}
\left(\mathbf{P 1 0} \mathbf{1 0}^{\prime \prime} \max _{\theta_{i}}\right. & \frac{\alpha}{2 \ln (2) \eta_{\mathrm{SE}}^{\max , 2}} \ln \left(1+\frac{\bar{\gamma}_{i, i} x_{i}^{2}}{\frac{1}{f_{\mathrm{c}}^{2}\left(\theta_{i}\right)}+\sum_{j=1, j \neq i}^{l} \bar{\gamma}_{i, \pi_{j}^{(i)}} x_{\pi_{j}^{(i)}}^{2}}\right)+\frac{1-\alpha}{P_{\mathrm{H}, \mathrm{tot}}^{\max , 2} \times} \\
& \left(\sum_{j=1}^{l} 0.75 h_{i, \pi_{j}^{(i)}}^{\mathrm{e}} f_{\mathrm{c}}\left(\theta_{i}\right) x_{\pi_{j}^{(i)}} \mathrm{V}_{\mathrm{t}} \ln \left(1+\sum_{j=1}^{l} h_{i, \pi_{j}^{(i)}}^{\mathrm{e}} f_{\mathrm{c}}\left(\theta_{i}\right) \frac{x_{\pi_{j}^{(i)}}}{I_{o}}\right)\right)
\end{aligned}
$$

subject to $\mathrm{C}^{\prime}: \theta_{i} \in\left[\max \left(\theta_{\min }, \psi_{i, i}\right), \theta_{\max }\right] \cap \mathcal{I}_{l} \quad, l \in\{0, \ldots, K\}$,

since $f_{\mathrm{c}}\left(\theta_{i}\right)$ is a non-increasing function, it follows that the objective of $\left(\mathbf{P} 10^{\prime \prime}\right)$ is non-increasing function and hence, its optimal solution $\theta_{i}^{(l)}$ can be expressed as

$$
\theta_{i}^{*(l)}= \begin{cases}\{\}, & \psi_{\pi_{l+1}^{(i)}}<\theta_{\min } \text { or } \psi_{\pi_{l}^{(i)}}>\theta_{\max } \\ \max \left(\theta_{\min }, \psi_{i, \pi_{l}^{(i)}}\right), & \text { otherwise. }\end{cases}
$$

Consequently, the optimal solution of (P10) can be expressed as

$$
\begin{gathered}
\theta_{i}^{*}=\arg \max _{\bar{\theta}_{i} \in \vartheta} \frac{\alpha}{2 \ln (2) \eta_{\mathrm{SE}}^{\max , 2}} \ln \left(1+\frac{\bar{\gamma}_{i, i} x_{i}^{2}}{\frac{1}{f_{\mathrm{c}}^{2}\left(\bar{\theta}_{i}\right)}+\sum_{j=1, j \neq i}^{K} \bar{\gamma}_{i, j} x_{j}^{2} \mathbf{1}\left(\bar{\theta}_{i} \geq \psi_{i, j}\right)}\right)+\frac{1-\alpha}{P_{\mathrm{H}, \mathrm{tot}}^{\max , 2}} \times \\
\left(\sum_{j=1}^{K} 0.75 h_{i, j}^{\mathrm{e}} f_{\mathrm{c}}\left(\bar{\theta}_{i}\right) \mathbf{1}\left(\bar{\theta}_{i} \geq \psi_{i, j}\right) x_{j} \mathrm{~V}_{\mathrm{t}} \ln \left(1+\sum_{j=1}^{K} h_{i, j}^{\mathrm{e}} f_{\mathrm{c}}\left(\bar{\theta}_{i}\right) \mathbf{1}\left(\bar{\theta}_{i} \geq \psi_{i, j}\right) \frac{x_{j}}{I_{o}}\right)\right),
\end{gathered}
$$

where $\vartheta=\bigcup_{l=0}^{K}\left\{\theta_{i}^{*(l)}\right\}$ which cannot be an empty set for a feasible (P10) (always guaranteed). It is worth to note that, for $\alpha=1$ this result reduces to $\theta_{i}^{*}=\min _{\bar{\theta}_{i} \in \vartheta} \bar{\theta}_{i}$, since the objective function of $(\mathbf{P} 10)$ is non-increasing in $\theta_{i}$, when $\alpha=1$, over the entire feasibility region.

It is worth to mention that when $\alpha=1$ and $P_{\mathrm{H}, \mathrm{th}}=0,(\mathbf{P} 6)$ and $(\mathbf{P} 1)$ become equivalent, similarly, (P9) and (P5) become equivalent. 
TABLE I: Simulation Parameters.

\begin{tabular}{|c|c|c|}
\hline$N_{0}=10^{-21} \mathrm{~W} / \mathrm{Hz}$ & $B_{\mathrm{v}}=20 \mathrm{MHz}$ & $H=3 \mathrm{~m}$ \\
\hline$P_{M}=450 \mathrm{~W}$ & $K=9$ & $h_{\mathrm{T}}=1 \mathrm{~m}$ \\
\hline$\phi_{\mathrm{A}}=60^{\circ}$ & $R_{\mathrm{PD}}^{\mathrm{d}}=R_{\mathrm{PD}}^{\mathrm{e}}=0.6 \mathrm{~A} / \mathrm{W}$ & $d_{\min }=0.5 \mathrm{~m}$ \\
\hline$\eta_{\phi}=429 \mathrm{~lm} / \mathrm{A}$ & $\kappa=1.11 \mathrm{~W} / \mathrm{A}^{2}$ & $\eta_{\mathrm{r}}=170 \mathrm{~lm} / \mathrm{W}$ \\
\hline$E_{\max }=1200 \mathrm{lux}$ & $E_{\min }=100 \mathrm{lux}$ & $\bar{\theta}=45^{\circ}$ \\
\hline$h_{\mathrm{c}}=0.25 \mathrm{~cm}$ & $r_{\mathrm{L}}=1 \mathrm{~cm}$ & $r_{\mathrm{D}}=0.2 \mathrm{~cm}$ \\
\hline
\end{tabular}

\section{Simulation Results}

The simulation setup consists of nine transmitters distributed over a square lattice on the ceiling of an indoor area at a height $H$ from the ground. It is assumed that user-cell association is done based on nearest distance, which is obviously translated to the square cell boundaries of side length $d_{\min }$ in the receivers plane as shown in Fig. 1. The nine users served by the system are distributed randomly such that each user is placed according to an uniform distribution within its corresponding transmitter square cell boundary in the receivers' plane. Moreover, the receivers-to-ground vertical separation is assumed to be $h_{\mathrm{T}}$, with all transmitters and receivers having horizontal orientation, with $\delta=0.5$. The average results presented in this work are calculated based on 1000 random users' locations realizations. The simulation parameters values are provided in Table $\mathrm{I}^{3}$, unless otherwise specified.

In the following simulations, we monitor the performances of different solutions, namely, SE maximization via TST (solution of (P1)), energy harvesting maximization via TST (solution of (P6), with $\alpha=0$ ), SE maximization via RST (solution of (P5)), energy harvesting maximization via RST (solution of (P9), with $\alpha=0$ ), projected waterfilling, which involves distributing the available power budget using the celebrated waterfilling algorithm while considering only direct channel gains, then projecting the solution on the feasibility region. In addition, we include in the performance comparison: SE maximization via joint average current allocation and FoVs tuning (TST-RST), which has two implementations: alternate optimization between either Alg. I or Alg. II with $\alpha=1$ and the solution of (P5), energy harvesting maximization via (TST-RST) which is implemented as an alternate execution between Alg. II with $\alpha=0$ and the solution of (P5). Furthermore, the performance comparison includes the global search algorithm provided

\footnotetext{
${ }^{3}$ The transmitting LEDs parameters values are based on XCree ${ }^{\circledR}$. XLamp-XM-L2 models datasheet [37]
} 


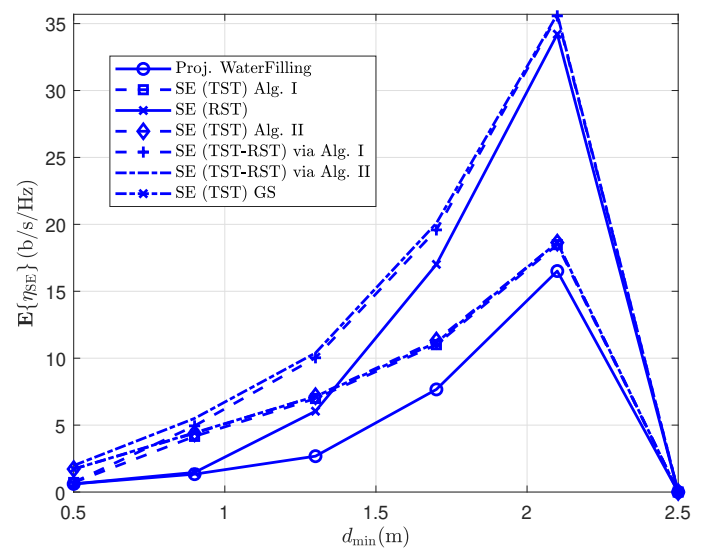

(a) Average SE vs $d_{\min }$

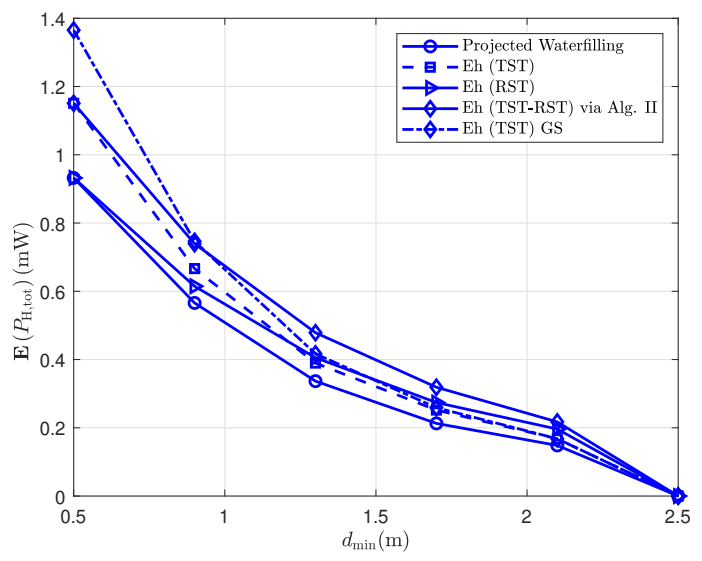

(b) Average $P_{\mathrm{H}, \text { tot }}$ vs $d_{\mathrm{min}}$

Fig. 4

in Matlab [38], which we use as a benchmark for all the TST strategies. The basic idea of this algorithm is that it generates a set of initial points in the feasibility region, then uses them as starting points for interior point method.

In the first simulation, we study the effect of resource allocation on the performance of SE and total harvested energy performance metrics when one of them is maximized versus the distance between neighboring cells. For TST strategies, we assume uniform FoV tuning where $\theta_{i}=\bar{\theta} \forall i$ while uniform average current allocation is assumed for RST such that $\boldsymbol{x}=$ $\boldsymbol{x}_{\mathbf{e q}}^{\max }$. We assume zero SE and energy harvesting performance for infeasible problem realizations in average calculation of these objectives. Moreover, we keep the per-user harvested power constraint inactive $(\mathrm{C} 4)$ by setting $P_{\mathrm{H}, \mathrm{th}}=0 \forall i$. The average SE performance versus $d_{\min }$ is shown in Fig. 4a, while the average total harvested energy performance is depicted in Fig. 4b. On the one hand, the SE performance of all the considered strategies exhibits a unimodal behavior with $d_{\min }$. For dense cell scenarios, i.e., as $d_{\min }$ decreases, the SE performance deteriorates due to increased interference. While for sparse cell scenarios, i.e., as $d_{\min }$ becomes relatively large, the feasibility region shrinks as the required $x_{\min }$ for minimum illumination requirement increases while the available power budget is kept fixed. Thus, the problem becomes infeasible due to insufficient power budget or conflict between the maximum and minimum illumination requirements, which results in a cutoff $d_{\min }$ as shown in Figs. 4a and 4b.

As for the relative performance between the considered solution strategies, it can be noticed 


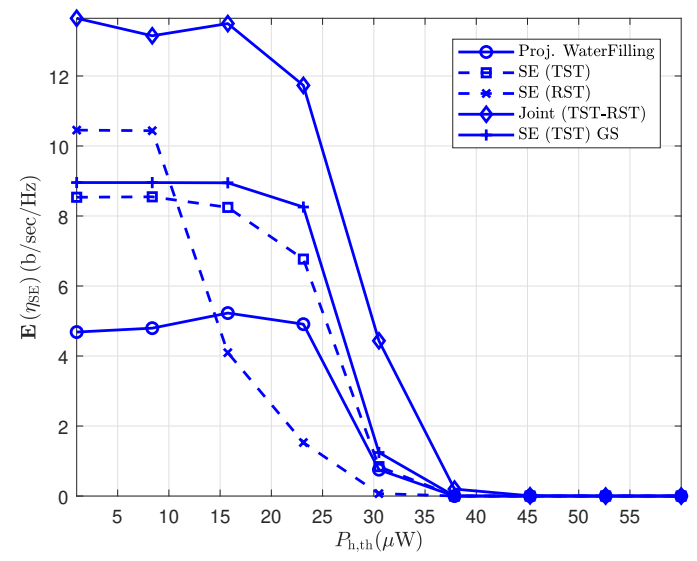

(a) Average $\mathrm{SE}$ vs $P_{\mathrm{H}, \mathrm{th}}$

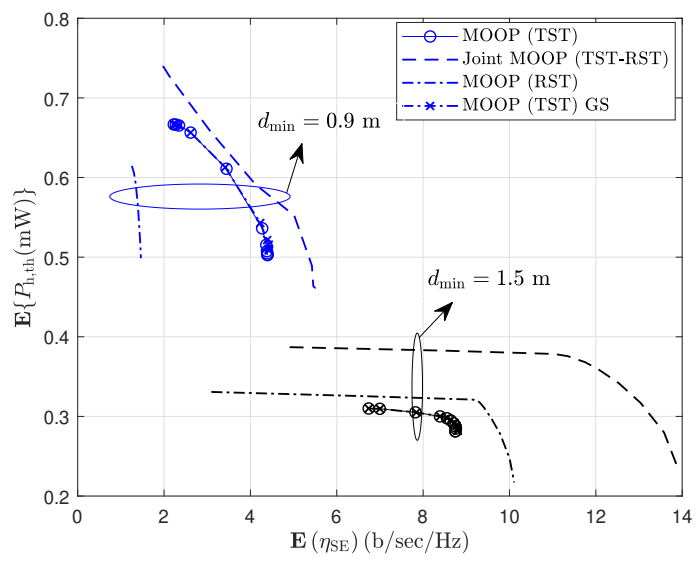

(b) Average $P_{\mathrm{H} \text {,tot }}$ vs Average SE tradeoff

Fig. 5

that TST strategies have superior performance compared with RST for small $d_{\text {min }}$, while the opposite effect can be seen for relatively large $d_{\text {min }}$ values. This effect owes to the limited receivers FoVs tunability range which makes them unable to reduce the strong interference suffered for small $d_{\mathrm{min}}$, which gives great importance to current allocation for interference management in this regime. Nonetheless, for larger $d_{\min }$ values RST gains its performance superiority as transmitters become at angles of incidence within the dynamic FoV range of the receivers, which allows them to suppress undesired interfering signals. Moreover, it can be noticed that the performance gap between joint (TST-RST) strategies and the maximum of separate (TST) and (RST strategies is relatively small for the extreme values of $d_{\text {min }}$ range while it is large for moderate $d_{\min }$ values. The added complexity of joint processing and hardware structure pays off only for certain range of $d_{\min }$ values.

On the other hand, the average total harvested power performance of all considered strategies gets worse as $d_{\min }$ gets larger, cf. Fig. $4 \mathrm{~b}$. This is expected since the users get further away from their associated transmitters, hence the effective areas of their receivers are exposed to smaller power densities. In similarity to SE performance results, the superiority of RST strategy shows clearly for large $d_{\text {min }}$ values, while TST strategies dominate in the small $d_{\min }$ regime. However, unlike SE performance results the performance gap between joint (TST-RST) and even the projected waterfilling solution which aims at maximizing SE in interference-free channels is not as severe. Moreover, it can be seen that the TST global search algorithm outperforms all 
the other strategies for small $d_{\min }$ values which suggests that there is a room to improve TST strategies and the alternating joint optimization strategies by choosing better initial points. It can be seen that the performance gap between all the considered strategies gets smaller as $d_{\min }$ increases.

In the second simulation, we study the effect of increasing the minimum individual harvested power constraint on the average SE performance for $d_{\min }=1.5 \mathrm{~m}$. It can be noticed that as $P_{\mathrm{H}, \mathrm{th}}$ increases the SE performance of optimized SE solution is not affected initially for both TST and RST strategies (C4 is not active yet). As $P_{\mathrm{H}, \text { th }}$ continues increasing, the SE performance deteriorates as the feasibility space gets tighter and the optimization solution satisfies C4 with equality for the TST strategy. It can be seen clearly that for small values of $P_{\mathrm{H}, \mathrm{th}}$, RST results in more improved SE efficiency than TST with relatively larger fixed FoV values that allows for significant interference. This effect is sustained till $P_{\mathrm{H}, \mathrm{th}}$ exceeds a certain limit beyond which TST strategy achieves better SE performance. This owes to the loss of superiority of RST by the virtue of raised level of interference at receivers enforced by $\mathrm{C} 4$. As $P_{\mathrm{H}, \mathrm{th}}$ keeps increasing, the probability of having more infeasible problems with zero contribution to the average SE performance increases.

In the third simulation, we study the effect of changing $d_{\min }$, on the inner-bound of the Pareto front between SE and the total harvested power achieved by MOOP via TST (solution of (P6)) in Fig. 5b. As $d_{\min }$ gets larger, the energy harvesting performance losses due to optimizing SE through TST becomes smaller. However for small $d_{\min }$, the SE performance losses due to energy harvesting optimization gets large. These effects are well illustrated by the behavior of SE-aimed TST strategy ( $\alpha=1)$, which tend to avoid interference by limiting power usage, and consequently deteriorates the harvested energy significantly while achieving small SE gains. Nevertheless, the energy harvesting-aimed TST strategy $(\alpha=0)$ will exhaust the whole power budget and generate interference that bans great possible SE gains for large $d_{\text {min }}$ values. As for the RST strategies, similar observations can be seen regarding the severity of performance loss in SE due to energy harvesting maximization and vice versa. The RST solution that targets SE maximization will reduce the receivers FoVs to their minimum values causing large energy harvesting performance losses for the small $d_{\min }$ regime. Contrarily, energy harvesting-aimed RST strategies will permit more interference levels at the receiver as they represent energy sources which prevents achieving potential SE gains in the large $d_{\min }$ regime. It can be observed that RST achieves better tradeoff for large the $d_{\text {min }}$ regime than TST, while this observation is 


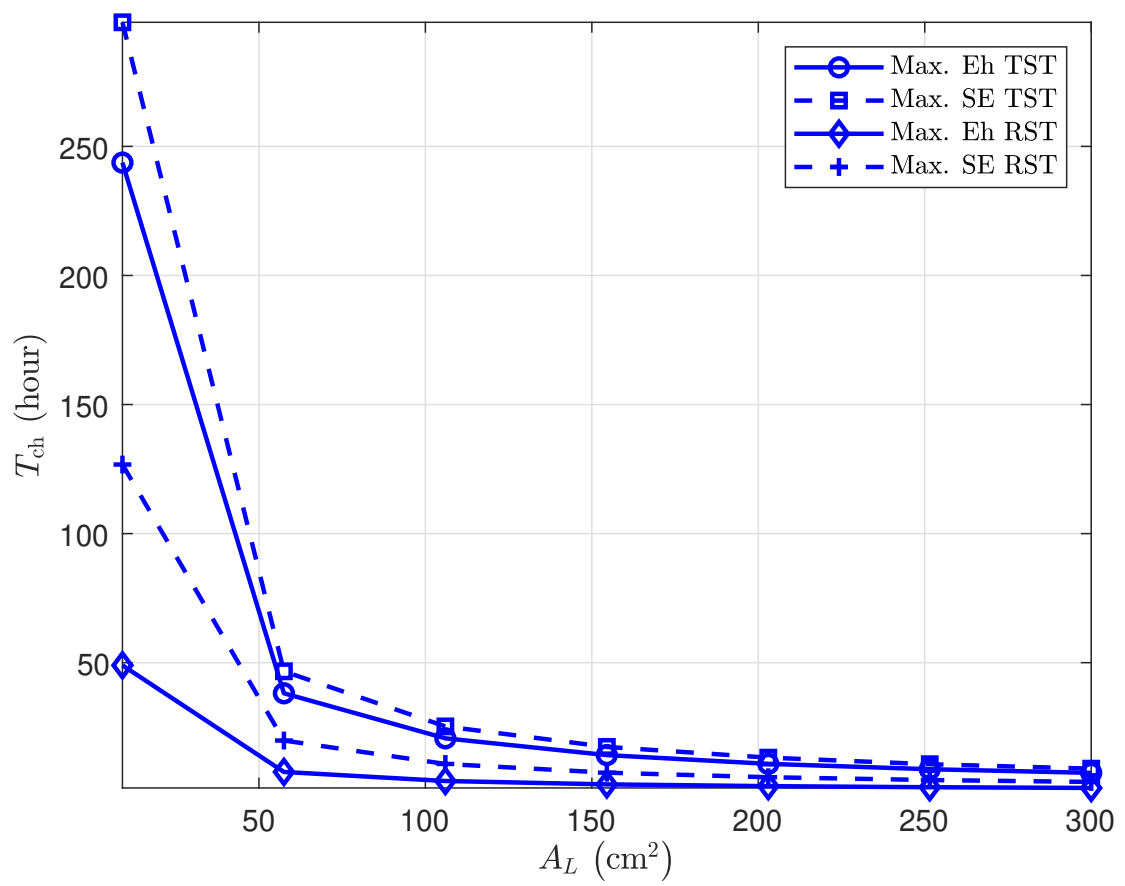

Fig. 6: Average charging time vs lens area

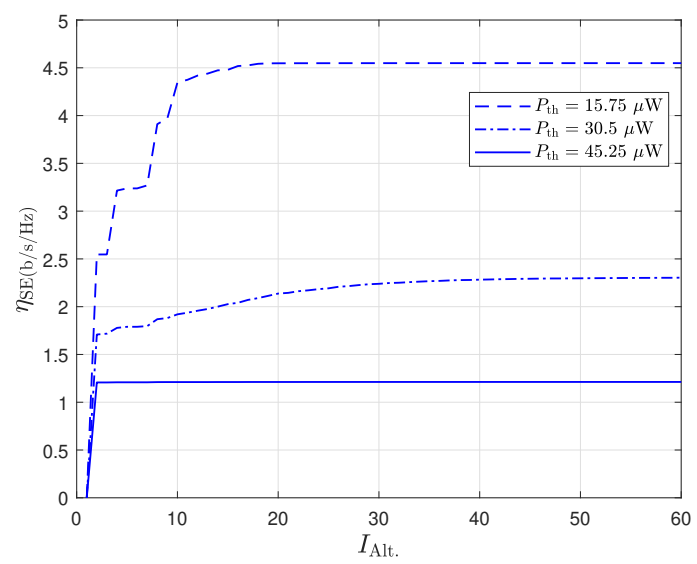

(a) Average SE vs $I_{\text {Alt }}$.

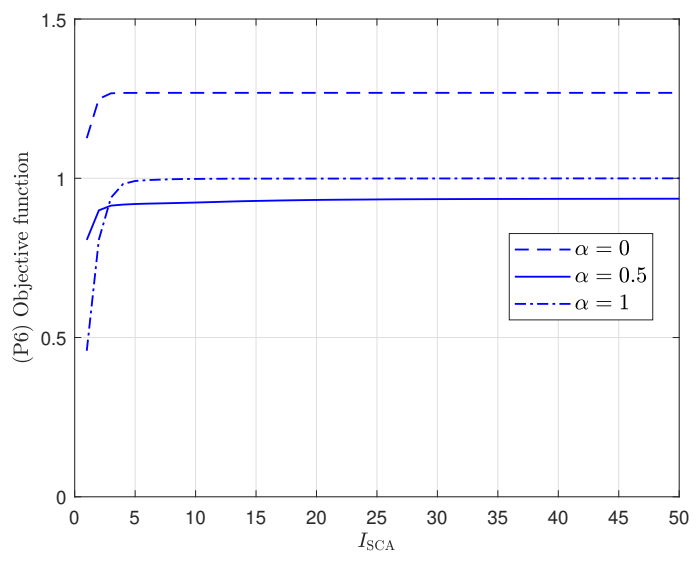

(b) Average (P6) objective function value vs $I_{\mathrm{SCA}}$

Fig. 7

reversed for the small $d_{\text {min }}$ regime.

In the fourth simulation, we study the effect of the optical receiver dimensions on the time required to charge a $100 \mathrm{mAh}$ battery when the two TST strategies: maximized energy harvesting TST (solution of (P6) with $\alpha=0$ ), Algorithm I in addition to the RST strategies aimed at 
maximizing SE and total harvested energy (solution of (P6) and solution of (P9) with $\alpha=0$, respectively). We increase the lens area from $9 \mathrm{~cm}^{2}$ to $300 \mathrm{~cm}^{2}$, which spans a wide range of portable devices, whilst keeping the dimensions ratio between the optical receiver components fixed. It can be observed from Fig. 6 that as the device size increases, the average required time to charge a certain battery decreases. As for the relative performance between the algorithms, it can be observed that total energy harvesting-driven TST and RST strategies outperform SE-driven ones, due to higher correlation between total harvested energy objective function with individual harvested energies than the correlation between SE objective and individual harvested energies. It can also be observed that energy harvesting driven RST strategy significantly outperform its TST counterpart, which makes FoV tuning crucial for individual energy harvesting performance.

In the last two simulations, we show the convergence behavior of the TST proposed strategies (Algorithm I and II). To this end, in Fig. 7a we show the average SE as alternation iteration number progresses for different values of $P_{\mathrm{th}}$, while in Fig. 7b we show the average value for the objective function of (P6) as iteration number of successive convex approximation procedure increases. In both simulations it can be seen that the average objective function value saturates within a reasonable number of iterations.

\section{CONCLUSION}

In this paper, we considered performance optimization of a multi-cell SLIPT system employing FoV-tunable receivers, for which we derived the optical concentration ratio in terms of FoV. We investigated the performance limits of this system through maximizing two of its design objectives, namely system overall SE, and total harvested energy under lighting constraints, and non-negligible interference assumption. We provided low complexity solutions for the SE maximization problem when the transmitters average current intensities and the receivers FoVs are tuned severally while individual energy harvesting constraint is imposed. In addition, we solved the MOOP involving overall SE and total harvested energy objectives through average current intensity allocation and FoV tuning. Moreover, we monitored the effect of optimizing each objective in terms of optimality losses on the other through extensive simulations. Simulation results highlighted the effect of cell deployment density on the effectiveness of the adopted tuning strategy. It was found that as cells deployment shift from density to sparsity, FoV tuning becomes more effective in contrast to average current allocation. This can be observed in the 
potential SE gains that can be attained under negligible individual energy harvesting constraints assumption for sparse deployments. Furthermore, the spacious Pareto frontiers shown in the simulation results shed the light on the severity of the tradeoff between the system overall SE and the total harvested energy given the considered degrees of freedom. As for future work, we recommend exploiting the area ratio of the receiver PD and solar cell to further improve the tradeoff relationship.

\section{APPENDIX A}

In this appendix we prove that $\eta_{\mathrm{MOOP}}(\boldsymbol{p})$ is a difference of two concave functions.

$$
\begin{gathered}
\eta_{\mathrm{MOOP}}(\boldsymbol{p})=\underbrace{\frac{\alpha}{2 \ln (2)}}_{\geq 0}(\underbrace{\sum_{i=1}^{K} \ln \left(1+\sum_{j=1}^{K} \tilde{\gamma}_{i, j} p_{j}\right)}_{\mathrm{T}_{1}}-\underbrace{\sum_{i=1}^{K} \ln \left(1+\sum_{j=1, j \neq i}^{K} \gamma_{i, j} p_{j}\right)}_{\mathrm{T}_{2}})+\underbrace{(1-\alpha)}_{\geq 0} P_{\mathrm{H}, \mathrm{tot}}(\boldsymbol{p}) \\
\tilde{P}_{\mathrm{H}, \mathrm{tot}}(\boldsymbol{p})=\underbrace{\sum_{i=1}^{K} \ln \left(\sum_{j=1}^{K} 0.75 h_{i, j}^{\mathrm{e}} \sqrt{p_{j}} \mathrm{~V}_{\mathrm{t}}\right)}_{\mathrm{T}_{3}}+\underbrace{\sum_{i=1}^{K} \ln \left(\ln \left(1+\sum_{j=1}^{K} h_{i, j}^{\mathrm{e}} \frac{\sqrt{p_{j}}}{I_{o}}\right)\right.}_{\mathrm{T}_{4}})
\end{gathered}
$$

The concavity of $T_{1}$ and $T_{2}$ in $\boldsymbol{p}$ follows from the fact that they are non-negative weighted sum of compositions of concave functions with affine terms in $\boldsymbol{p}$. As for $P_{\mathrm{H}, \text { tot }}(\boldsymbol{p})$, we show its concavity as follows

$P_{\mathrm{H}, \mathrm{tot}}(\boldsymbol{p})=0.75 V_{\mathrm{t}} \sum_{i=1}^{K}\left(\sum_{l=1}^{K} h_{i, l}^{\mathrm{e}} \sqrt{p_{l}}\right) \ln \left(1+\sum_{j=1}^{K} h_{i, j}^{\mathrm{e}} \sqrt{p_{j}} / I_{\mathrm{o}}\right) \triangleq 0.75 V_{\mathrm{t}} \sum_{i=1}^{K} \sum_{l=1}^{K} f_{i, l}(\boldsymbol{p})$,

where $f_{i, l}(\boldsymbol{p})=h_{i, l} \sqrt{p_{l}} \ln \left(1+\sum_{j=1}^{K} \frac{h_{i, j}^{\mathrm{e}}}{I_{\mathrm{o}}} \sqrt{p_{j}}\right)$. The concavity of $P_{\mathrm{H}, \text { tot }}(\boldsymbol{p})$ follows from the concavity of $f_{i, l}(\boldsymbol{p})$. By restricting the input of $f_{i, l}(\boldsymbol{p})$ to an arbitrary line $\boldsymbol{p}=\boldsymbol{p}_{\boldsymbol{o}}+t \boldsymbol{p}^{\prime}$, such that $\boldsymbol{p}_{\boldsymbol{o}}$ and $\boldsymbol{p}_{\boldsymbol{o}}+t \boldsymbol{p}^{\prime}$ lie in the feasibility space of (P6) (all their elements are positive), and investigating the concavity of $f_{i, l}\left(\boldsymbol{p}_{\boldsymbol{o}}+t \boldsymbol{p}^{\prime}\right)$ in $t[36,3.1]$. We find the second derivative of $f_{i, l}\left(\boldsymbol{p}_{\boldsymbol{o}}+t \boldsymbol{p}^{\prime}\right)$ with respect to $t$ as follows:

$$
\begin{aligned}
& \frac{\partial^{2} f_{i, l}}{\partial t^{2}}=\frac{\partial^{2}}{\partial t^{2}} h_{i, l} \sqrt{p_{o, l}+t p_{l}^{\prime}} \ln \left(1+\sum_{j=1}^{K} \frac{h_{i, j}^{\mathrm{e}}}{I_{\mathrm{o}}} \sqrt{p_{o, j}+t p_{j}^{\prime}}\right) \\
& =\frac{p_{l}^{\prime}\left(\sum_{k=1}^{n} \frac{h_{k} p_{k}^{\prime}}{2 \sqrt{t p_{k}^{\prime}+p_{o, k}}}\right)}{\sqrt{t p_{l}^{\prime}+p_{o, l}}\left(\sum_{k=1}^{n} h_{k} \sqrt{t p_{k}^{\prime}+p_{o, k}}+1\right)}-\frac{\sqrt{t p_{l}^{\prime}+p_{o, l}} \sum_{k=1}^{n} \frac{h_{k} p_{k}^{\prime 2}}{4\left(t p_{k}^{\prime}+p_{o, k}\right)^{3 / 2}}}{\sum_{k=1}^{n} h_{k} \sqrt{t p_{k}^{\prime}+p_{o, k}}+1}
\end{aligned}
$$




$$
-\frac{\sqrt{t p_{l}^{\prime}+p_{o, l}}\left(\sum_{k=1}^{n} \frac{h_{k} p_{k}^{\prime}}{2 \sqrt{t p_{k}^{\prime}+p_{o, k}}}\right)^{2}}{\left(\sum_{k=1}^{n} h_{k} \sqrt{t p_{k}^{\prime}+p_{o, k}}+1\right)^{2}}-\frac{p_{l}^{\prime 2} \ln \left(\sum_{k=1}^{n} h_{k} \sqrt{t p_{k}^{\prime}+p_{o, k}}+1\right)}{4\left(t p_{l}^{\prime}+p_{o, l}\right)^{3 / 2}} .
$$

By using $\frac{x-1}{x} \leq \ln (1+x) \quad \forall x>0$ to lower bound the logarithmic term, the following upper bound is obtained on $\frac{\partial^{2} f_{i, l}}{\partial t^{2}}$,

$$
\begin{gathered}
\frac{\partial^{2} f_{i, l}}{\partial t^{2}} \leq \frac{p_{l}^{\prime}\left(\sum_{k=1}^{n} \frac{h_{k} p_{k}^{\prime}}{2 \sqrt{t p_{k}^{\prime}+p_{o, k}}}\right)}{\sqrt{t p_{l}^{\prime}+p_{o, l}}\left(\sum_{k=1}^{n} h_{k} \sqrt{t p_{k}^{\prime}+p_{o, k}}+1\right)}-\frac{\sqrt{t p_{l}^{\prime}+p_{o, l}} \sum_{k=1}^{n} \frac{h_{k} p_{k}^{\prime 2}}{4\left(t p_{k}^{\prime}+p_{o, k}\right)^{3 / 2}}}{\sum_{k=1}^{n} h_{k} \sqrt{t p_{k}^{\prime}+p_{o, k}}+1} \\
-\frac{\sqrt{t p_{l}^{\prime}+p_{o, l}}\left(\sum_{k=1}^{n} \frac{h_{k} p_{k}^{\prime}}{2 \sqrt{t p_{k}^{\prime}+p_{o, k}}}\right)^{2}}{\left(\sum_{k=1}^{n} h_{k} \sqrt{t p_{k}^{\prime}+p_{o, k}}+1\right)^{2}}-\frac{p_{k=1}^{n} h_{k} \sqrt{t p_{k}^{\prime}+p_{o, k}}}{4\left(t p_{l}^{\prime}+p_{o, l}\right)^{3 / 2}\left(\sum_{k=1}^{n} h_{k} \sqrt{t p_{k}^{\prime}+p_{o, k}}+1\right)} .
\end{gathered}
$$

By combining the first two terms together, the last two terms together and unifying the denominators, the right hand side (R.H.S) reduces to

$$
\begin{gathered}
-\frac{4\left(t p_{l}^{\prime}+p_{o, l}\right)\left(\sum_{k=1}^{n} h_{k} \sqrt{t p_{k}^{\prime}+p_{o, k}}+1\right) \underbrace{\sum_{k=1}^{n} \frac{h_{k} p_{k}^{\prime 2}\left(t p_{l}^{\prime}+p_{o, l}\right)-0.5 h_{k} p_{l}^{\prime} p_{k}^{\prime}\left(t p_{k}^{\prime}+p_{o, k}\right)}{4\left(t p_{k}^{\prime}+p_{o, k}\right)^{3 / 2}}}_{T_{3}}}{4\left(t p_{l}^{\prime}+p_{o, l}\right)^{3 / 2}\left(\sum_{k=1}^{n} h_{k} \sqrt{t p_{k}^{\prime}+p_{o, k}}+1\right)^{2}} \\
-\frac{4\left(t p_{l}^{\prime}+p_{o, l}\right)^{2}\left(\sum_{k=1}^{n} \frac{h_{k} p_{k}^{\prime}}{2 \sqrt{t p_{k}^{\prime}+p_{o, k}}}\right)^{2} \underbrace{-p_{l}^{\prime 2} \sum_{k=1}^{n} h_{k} \sqrt{t p_{k}^{\prime}+p_{o, k}}\left(\sum_{k=1}^{n} h_{k} \sqrt{t p_{k}^{\prime}+p_{o, k}}+1\right)}_{T_{3}^{\prime}}}{4\left(t p_{l}^{\prime}+p_{o, l}\right)^{3 / 2}\left(\sum_{k=1}^{n} h_{k} \sqrt{t p_{k}^{\prime}+p_{o, k}}+1\right)^{2}}
\end{gathered}
$$

By combining $T_{2}$ and $T_{2}^{\prime}$, the R.H.S of (24) becomes

$$
\begin{aligned}
& \sum_{k=1}^{n} h_{k} \underbrace{\frac{p_{k}^{\prime 2}\left(t p_{l}^{\prime}+p_{o, l}\right)^{2}-0.5 p_{l}^{\prime} p_{k}^{\prime}\left(t p_{k}^{\prime}+p_{o, k}\right)\left(t p_{l}^{\prime}+p_{o, l}\right)+p_{l}^{\prime 2}\left(t p_{k}^{\prime}+p_{o, k}\right)^{2}}{\left(t p_{k}^{\prime}+p_{o, k}\right)^{3 / 2}}}_{T_{3}^{\prime \prime}} \\
& 4\left(t p_{l}^{\prime}+p_{o, l}\right)^{3 / 2}\left(\sum_{k=1}^{n} h_{k} \sqrt{t p_{k}^{\prime}+p_{o, k}}+1\right) \\
& -\frac{\left(t p_{l}^{\prime}+p_{o, l}\right)^{2}\left(\sum_{k=1}^{n} \frac{h_{k} p_{k}^{\prime}}{2 \sqrt{t p_{k}^{\prime}+p_{o, k}}}\right)^{2}}{\left(t p_{l}^{\prime}+p_{o, l}\right)^{3 / 2}\left(\sum_{k=1}^{n} h_{k} \sqrt{t p_{k}^{\prime}+p_{o, k}}+1\right)^{2}} .
\end{aligned}
$$


By completing the square in the numerator of $T_{3}^{\prime \prime}$, the R.H.S of (24) is further simplified to

$$
\frac{-\sum_{k=1}^{n} h_{k} \frac{\left(p_{k}^{\prime}\left(t p_{l}^{\prime}+p_{o, l}\right)-p_{l}^{\prime}\left(t p_{k}^{\prime}+p_{o, k}\right)\right)^{2}+1.5 p_{l}^{\prime} p_{k}^{\prime}\left(t p_{k}^{\prime}+p_{o, k}\right)\left(t p_{l}^{\prime}+p_{o, l}\right)}{\left(t p_{k}^{\prime}+p_{o, k}\right)^{3 / 2}}}{4\left(t p_{l}^{\prime}+p_{o, l}\right)^{3 / 2}\left(\sum_{k=1}^{n} h_{k} \sqrt{t p_{k}+p_{o, k}}+1\right)}-\frac{\left(t p_{l}^{\prime}+p_{o, l}\right)^{\frac{1}{2}}\left(\sum_{k=1}^{n} \frac{h_{k} p_{k}}{2 \sqrt{t p_{k}+p_{o, k}}}\right)^{2}}{\left(\sum_{k=1}^{n} h_{k} \sqrt{t p_{k}+p_{o, k}}+1\right)^{2}} \leq \text {, }
$$

which proves the concavity of $P_{\mathrm{H}, \text { tot }}(\boldsymbol{p})$ and completes the proof.

\section{REFERENCES}

[1] A. M. Abdelhady, O. Amin, B. Shihada, and M. S. Alouini, "On the optimization of multi-cell SLIPT systems," in IEEE Globecom, Abu Dhabi, Dec. 2018, pp. 1-6.

[2] M. A. Khalighi and M. Uysal, "Survey on free space optical communication: A communication theory perspective," IEEE Commun. Surveys Tuts., vol. 16, no. 4, pp. 2231-2258, Jun. 2014.

[3] S. Dimitrov and H. Haas, Principles of LED light communications: towards networked Li-Fi. Cambridge University Press, 2015.

[4] P. H. Pathak, X. Feng, P. Hu, and P. Mohapatra, "Visible light communication, networking, and sensing: A survey, potential and challenges," IEEE Commun. Surveys Tuts., vol. 17, no. 4, pp. 2047-2077, Fourthquarter 2015.

[5] C.-X. Wang, F. Haider, X. Gao, X.-H. You, Y. Yang, D. Yuan, H. Aggoune, H. Haas, S. Fletcher, and E. Hepsaydir, "Cellular architecture and key technologies for 5G wireless communication networks," IEEE Commun. Mag., vol. 52, no. 2, pp. 122-130, Feb. 2014.

[6] J. Gancarz, H. Elgala, and T. D. C. Little, "Impact of lighting requirements on VLC systems," IEEE Commun. Mag., vol. 51, no. 12, pp. 34-41, December 2013.

[7] W.-H. Shin, S.-H. Yang, D.-H. Kwon, and S.-K. Han, "Self-reverse-biased solar panel optical receiver for simultaneous visible light communication and energy harvesting," Optics express, vol. 24, no. 22, pp. A1300-A1305, 2016.

[8] H. Haas, S. Videv, S. Das, J. Fakidis, and H. Stewart, "Solar cell receiver free-space optical for 5g backhaul," in Optical Fiber Communication Conference. Optical Society of America, 2019, pp. M3G-2.

[9] P. Kamalinejad, C. Mahapatra, Z. Sheng, S. Mirabbasi, V. C. Leung, and Y. L. Guan, "Wireless energy harvesting for the internet of things," IEEE Commun. Mag., vol. 53, no. 6, pp. 102-108, 2015.

[10] H. G. Sandalidis, A. Vavoulas, T. A. Tsiftsis, and N. Vaiopoulos, "Illumination, data transmission, and energy harvesting: the threefold advantage of VLC," Applied optics, vol. 56, no. 12, pp. 3421-3427, 2017.

[11] A. M. Abdelhady, O. Amin, A. Chaaban, B. Shihada, and M. S. Alouini, "Spectral-efficiency - illumination pareto front for energy harvesting enabled VLC system.” [Online]. Available: http://hdl.handle.net/10754/626362

[12] T. Rakia, H. C. Yang, F. Gebali, and M. S. Alouini, "Optimal design of dual-hop VLC/RF communication system with energy harvesting," IEEE Commun. Lett., vol. 20, no. 10, pp. 1979-1982, Oct. 2016.

[13] M. R. Zenaidi, Z. Rezki, M. Abdallah, K. A. Qaraqe, and M. S. Alouini, "Achievable rate-region of VLC/RF communications with an energy harvesting relay," in IEEE Global Commun. Conf. (GLOBECOM), Dec. 2017, pp. 17.

[14] A. M. Abdelhady, O. Amin, A. Chaaban, and M. S. Alouini, "Resource allocation for outdoor visible light communications with energy harvesting capabilities," in IEEE Globecom Workshops (GC Wkshps), Singapore, Dec. 2017, pp. 1-6. 
[15] Y. Li, N. Huang, J. Wang, Z. Yang, and W. Xu, "Sum rate maximization for VLC systems with simultaneous wireless information and power transfer," IEEE Photon. Technol. Lett., vol. 29, no. 6, Mar. 2017.

[16] P. D. Diamantoulakis, G. K. Karagiannidis, and Z. Ding, "Simultaneous lightwave information and power transfer (SLIPT)," IEEE Trans.Green Commun. Netw., Doi: 10.1109/TGCN.2018.2818325 2018.

[17] Y. Liu, H. Y. Chen, K. Liang, C. W. Hsu, C. W. Chow, and C. H. Yeh, "Visible light communication using receivers of camera image sensor and solar cell,” IEEE Photon. J., vol. 8, no. 1, pp. 1-7, Feb. 2016.

[18] G. Pan, P. D. Diamantoulakis, Z. Ma, Z. Ding, and G. K. Karagiannidis, "Simultaneous Lightwave Information and Power Transfer: Policies, Techniques, and Future Directions," IEEE Access, vol. 7, pp. 28 250-28 257, Feb. 2019.

[19] A. G. Al-Ghamdi and J. M. H. Elmirghani, "Performance evaluation of a triangular pyramidal fly-eye diversity detector for optical wireless communications," IEEE Commun. Mag., vol. 41, no. 3, pp. 80-86, Mar. 2003.

[20] K. Park, H. M. Oubei, W. G. Alheadary, B. S. Ooi, and M. Alouini, "A novel mirror-aided non-imaging receiver for indoor $2 \times 2$ MIMO-visible light communication systems," IEEE Trans. Wireless Commun., vol. 16, no. 9, pp. 5630-5643, Sept. 2017.

[21] C. Chen, W. Zhong, H. Yang, S. Zhang, and P. Du, "Reduction of SINR fluctuation in indoor multi-cell VLC systems using optimized angle diversity receiver," J. Lightw. Technol., vol. 36, no. 17, pp. 3603-3610, Sept. 2018.

[22] M. S. Rea, The IESNA lighting handbook: reference \& application. IESNA, 2000.

[23] Z. Wang, D. Tsonev, S. Videv, and H. Haas, "On the design of a solar-panel receiver for optical wireless communications with simultaneous energy harvesting," IEEE J. Sel. Areas Commun., vol. 33, no. 8, pp. 1612-1623, Aug. 2015.

[24] H. Ren and W. Shin-Tson, Introduction to Adaptive Lenses. John Wiley \& Sons, 2012.

[25] J. M. Kahn and J. R. Barry, “Wireless infrared communications,” Proc. IEEE, vol. 85, no. 2, pp. 265-298, Feb. 1997.

[26] W. T. Welford and R. Winston, High collection nonimaging optics. Academic Press Inc., 1989.

[27] B. E. Saleh and M. C. Teich, Fundamentals of photonics. John Wiley \& Sons, 2019.

[28] A. Lapidoth, S. M. Moser, and M. Wigger, "On the capacity of free-space optical intensity channels," IEEE Trans. Inf. Theory, vol. 55, no. 10, pp. 4449-4461, Oct. 2009.

[29] T. Marzetta, E. Larsson, Y. Hong, and H. Ngo, Fundamentals of Massive MIMO. Cambridge University Press, 2016.

[30] E. Lorenzo, Solar electricity: engineering of photovoltaic systems. Earthscan/James \& James, 1994.

[31] A. M. Abdelhady, O. Amin, A. Chaaban, and M. S. Alouini, "Downlink resource allocation for multichannel TDMA visible light communications," in 2016 IEEE Global Conf. Signal Inform. Process. (GlobalSIP), Greater Washington, D.C., USA, Dec. 2016, pp. 1-5.

[32] T. Lipp and S. Boyd, "Variations and extension of the convex-concave procedure," Optimization and Engineering, vol. 17, no. 2, pp. 263-287, 2016.

[33] M. Grant and S. Boyd, "CVX: Matlab software for disciplined convex programming, version 2.1," http://cvxr.com/cvx, Mar. 2014

[34] I. Bang, S. M. Kim, and D. K. Sung, "Adaptive multiuser scheduling for simultaneous wireless information and power transfer in a multicell environment," IEEE Transactions on Wireless Communications, vol. 16, no. 11, pp. 7460-7474, Nov. 2017.

[35] O. Amin, E. Bedeer, M. H. Ahmed, and O. A. Dobre, "Energy efficiency-spectral efficiency tradeoff: A multiobjective optimization approach,” IEEE Transactions on Vehicular Technology, vol. 65, no. 4, pp. 1975-1981, April 2016.

[36] S. Boyd and L. Vandenberghe, Convex optimization. Cambridge university press, 2004.

[37] "Product family datasheet," CREE, rev. 6K.

[38] Z. Ugray, L. Lasdon, J. Plummer, F. Glover, J. Kelly, and R. Martí, "Scatter search and local nlp solvers: A multistart framework for global optimization,” INFORMS Journal on Computing, p. 2007. 